\title{
Article \\ The Axial Anomaly in Lorentz Violating Theories: Towards the Electromagnetic Response of Weakly Tilted Weyl Semimetals
}

\author{
Andrés Gómez ${ }^{1,+} \mathbb{D}$ and Luis Urrutia ${ }^{2, *,+} \mathbb{D}$ \\ 1 Facultad de Ciencias, Universidad Nacional Autónoma de México, Circuito Exterior, C.U., \\ Ciudad de México 04510, Mexico; andresgz@ciencias.unam.mx \\ 2 Instituto de Ciencias Nucleares, Universidad Nacional Autónoma de México, Circuito Exterior, C.U., \\ Ciudad de México 04510, Mexico \\ * Correspondence: urrutia@nucleares.unam.mx \\ + These authors contributed equally to this work.
}

check for updates

Citation: Gómez, A.; Urrutia, L. The Axial Anomaly in Lorentz Violating Theories: Towards the Electromagnetic Response of Weakly Tilted Weyl Semimetals. Symmetry 2021, 13, 1181. https://doi.org/ $10.3390 /$ sym 13071181

Academic Editor: Matthew Mewes

Received: 3 June 2021

Accepted: 23 June 2021

Published: 30 June 2021

Publisher's Note: MDPI stays neutral with regard to jurisdictional claims in published maps and institutional affiliations.

Copyright: (c) 2021 by the authors. Licensee MDPI, Basel, Switzerland. This article is an open access article distributed under the terms and conditions of the Creative Commons Attribution (CC BY) license (https:// creativecommons.org/licenses/by/ $4.0 /)$.

\begin{abstract}
Using the path integral formulation in Euclidean space, we extended the calculation of the abelian chiral anomalies in the case of Lorentz violating theories by considering a new fermionic correction term provided by the standard model extension, which arises in the continuous Hamiltonian of a weakly tilted Weyl semimetal, and whose cones have opposite tilting. We found that this anomaly is insensitive to the tilting parameter, retaining its well-known covariant form. This independence on the Lorentz violating parameters is consistent with other findings reported in the literature. The initially imposed gauge invariant regularization was consistently recovered at the end of the calculation by the appearance of highly non-trivial combinations of the covariant derivatives, which ultimately managed to give only terms containing the electromagnetic tensor. We emphasize that the value of the anomaly with an arbitrary parameter is not automatically related to the effective action describing the electromagnetic response of such materials.
\end{abstract}

Keywords: Lorentz invariance violation; Weyl semimetals; abelian chiral anomaly; path integral approach; effective electromagnetic response

\section{Introduction}

The appearance of fermionic excitations in the continuum Hamiltonians of condensed matter materials, which naturally violate some spacetime symmetries and incorporate contributions similar to those in the fermionic sector of the standard model extension (SME) [1,2], has created the possibility of applying many of the techniques already developed in the search for a fundamental Lorentz violation in the interactions of high energy physics to this area. This also occurs in the effective electromagnetic response of magnetoelectic materials, for example, which also reproduce some of the terms included in the electromagnetic sector of the SME $[3,4]$.

Macroscopic electromagnetic transport properties in condensed matter are often calculated using the Kubo formula [5] or the semiclassical Boltzmann approach [6]. Recently, the rise of topological phases of matter has promoted the use of anomaly calculations for these purposes. The topological properties of anomalies have long been recognized in high energy physics and their presence is expected to provide a macroscopic understanding of the underlying topological properties in the electronic design of these materials. Such an approach has been particularly fruitful in the case of Weyl semimetals (WSMs) whose electronic Hamiltonians naturally include some of the Lorentz invariance violating (LIV) terms considered in the fermionic sector of the standard model extension (SME) [7]. Nevertheless, in this case, the LIV parameters need not be highly suppressed, since they are determined by the electronic structure of the material and subjected to experimental determination. Weyl semimetals were first theoretically predicted in pyrochlore iridates (such as $\mathrm{Y}_{2} \mathrm{Ir}_{2} \mathrm{O}_{7}$ ) in 2011 [8] and experimentally discovered in TaAs four years later [9-13]. Their low energy 
excitations near the Fermi energy are described by Weyl fermions whose band structure is characterized by an even number of gapless Weyl nodes with opposite chiralities, which are the only points where the valence band touches the conduction band. This pair-wise characteristic is a consequence of the Nielsen-Ninomiya theorem [14] and the stability of the Weyl cones is guaranteed by the breaking of time reversal and/or inversion symmetries. Each pair of nodes can present a separation in energy and/or momentum in the Brillouin zone. Fixing one of the momenta in the Brillouin zone, say $k_{z}$, the dispersion relation of the Weyl fermions is $E(\mathbf{k})= \pm \sqrt{k_{x}^{2}+k_{y}^{2}}$ for the energy spectrum near the nodes. This surface describes a cone with an apex at the origin and axis perpendicular to the $\left(k_{x}-k_{y}\right)$ plane where we fix the Fermi energy touching the cone just in the apex.

A simple model of a WSM consisting of two Weyl nodes is described by the fermionic action:

$$
S=\int d^{4} x\left(i \bar{\Psi}\left(\gamma^{\mu} \partial_{\mu}+i b_{\mu} \gamma^{\mu} \gamma^{5}\right) \Psi\right)
$$

where $b_{\mu}$ signals a Lorentz violating term which accounts for the separation of the Weyl nodes in energy $\left(b_{0}\right)$ and in momentum $(\mathbf{b})$. Adding an additional Lorentz violating term in the derivative term of the action (1) makes it possible to give an inclination to the cone axis, thus defining a tilted WSM. For small inclinations (small tilting), we keep the point-like Fermi surface and these materials are called Type-I WSMs. By increasing the tilting, we arrive at a point where the conical surface becomes tangent to the $\left(k_{x}-k_{y}\right)$ plane. A further increment will produce an intersection of the conical surface with the Fermi energy plane creating what are called the electron and hole pockets, which takes us out from the point-like Fermi surface. This second possibility gives rise to Type II WSMs [15,16].

Among the novel transport properties of WSMs, we find the chiral magnetic effect, whereby a ground state dissipationless current proportional to an applied magnetic field is generated in the bulk of a WSM with broken inversion symmetry [17]. This yields a conductivity proportional to the magnetic field, or equivalently, to a resistivity that decreases with an increasing magnetic field. This phenomenon, dubbed as negative magnetoresistance, was predicted in Ref. [14] and was experimentally observed in Ref. [18]. WSMs also exhibit the anomalous Hall effect, characterized by Hall conductivity proportional to the separation of the Weyl nodes in momentum [19-22]. For a review of WSMs, see, for example, Refs. [23-25].

The appearance of Weyl fermions quasi-particles in WSMs naturally introduces the issue of anomalies when gauge invariance is demanded via the minimal coupling with external electromagnetic fields which is required to probe the electromagnetic response of such materials [26]. In this way, the chiral current $J_{\mu}^{5}=\bar{\Psi} \gamma^{\mu} \gamma^{5} \Psi$ is not conserved, yielding the abelian version of the chiral anomaly:

$$
\partial^{\mu} J_{\mu}^{5}=-\frac{e^{2}}{16 \pi^{2}} \epsilon^{\mu \nu \alpha \beta} F_{\mu \nu} F_{\alpha \beta}=+\frac{e^{2}}{2 \pi^{2}} \mathbf{E} \cdot \mathbf{B},
$$

in the Lorentz covariant case [27,28]. Here we follow the conventions of Ref. [29] with $\epsilon^{0123}=\epsilon^{1234}=+1$. In the path integral approach, the effective macroscopic electromagnetic action is obtained by introducing the electromagnetic coupling in (1) and subsequently trading the fermionic term $\bar{\Psi} b_{\mu} \gamma^{\mu} \gamma_{5} \Psi$ by an electromagnetic contribution arising from the Jacobian of the chiral transformation, which eliminates the $b_{\mu}$ term from the action [21]. Following this idea, the Fujikawa prescription to calculate the chiral anomalies [30,31] has been extensively used for these purposes [21,22].

The relevance of the tilting of the cones in the transport properties of WSMs was first reported in Ref. [15]. Subsequent works aiming to understand the role of the chiral anomaly in this process were based on the semiclassical Boltzman approach. Here, the contribution of the anomaly was identified through the term $\mathbf{E} \cdot \mathbf{B}$ which appears as a factor of the Berry phase in the equation of motion of the momentum of the wave packet, which is finally solved in terms of the external electromagnetic fields [32,33]. 
In this work, we take the first steps in calculating the effective electromagnetic action of an isotropic tilted Type I WSM, by considering a model with a single pair of Weyl points with tilting in opposite directions. Using the path integral approach, we deal with the calculation of the anomaly corresponding to the modified axial current responsible for the tilting of the cones around each node, which amounts to discarding the effect of the $b_{\mu}$ contribution in a first approximation. In this restricted setting, our work is similar to the calculation of the anomaly presented in Ref. [34], however, we find some important differences arising from the non-commutativity of the operators involved in the calculation, which we report in Section 6. We only consider the corrections to the anomaly which are linear and quadratic in the tilting parameter.

This paper is organized as follows. In Section 2, we define our model and provide a brief explanation of WSMs, setting the notation and conventions. Section 3 deals with a review of the axial anomaly in the path integral approach when going to Euclidean space. The modified axial current is calculated and the basic operators required for the implementation of the Fujikawa method are derived. We then proceed to the first order calculation (linear in the tilting parameter) in Section 4 , followed by the second order calculation (quadratic in the tilting parameter) in Section 5. We conclude with a summary and a discussion of the results in Section 6. In this section, we also make a comparison with previous findings in the literature. The Appendix A summarizes some useful relations used in the calculations.

\section{The Modified Dirac Lagrangian}

In this article, we consider the Lagrangian:

$$
\mathcal{L}=\bar{\Psi}(x) i \mathbb{D} \Psi(x)=i \bar{\Psi}(x)\left(\gamma^{\mu} D_{\mu}+\gamma^{0} \gamma^{5} v^{i} D_{i}\right) \Psi(x),
$$

which describes the dynamics of a massless spinor field $\Psi(x)$, coupled with an external electromagnetic field $A_{\mu}$ through the covariant derivative $D_{\mu}=\partial_{\mu}-i e A_{\mu}$. The standard gamma matrices $\gamma^{\mu}$ would ensure the Lorentz invariance of the Lagrangian if it were not for the presence of the second term. This term contains the pseudoscalar matrix $\gamma^{5}=i \gamma^{0} \gamma^{1} \gamma^{2} \gamma^{3}$ and a Lorentz violating parameter $v^{i}, i=1,2,3$, which we consider as the components of a spacetime vector $\mathbf{v}$. In this way, we keep invariance under rotations. For $\mathbf{v}=0$, we recover the usual Dirac Lagrangian for massless spinors. As is usual, $\bar{\Psi}(x)=\Psi^{\dagger} \gamma^{0}$.

The physical motivations for taking the modified Lagrangian (3) can be seen from two branches of physics. On one hand, in the description of Weyl semimetals, $\mathbf{v}$ parametrizes a tilt of the cones in a system with a pair of Weyl nodes. This parameter, which is determined by the electronic structure of the material, could in principle take any value, as Lorentz invariance is effectively broken into condensed matter. On the other hand, in the context of quantum field theory, this term probes the possibility of a fundamental breaking of Lorentz invariance. As this possibility has not been found in the many high-precision experiments already performed, we must consider this parameter as very small in this setting. Indeed, the Lagrangian (3) is a particular case of:

$$
\mathcal{L}=\bar{\Psi}(x)\left(i \Gamma^{\mu} \partial_{\mu}-M\right) \Psi(x)
$$

which arises in the study of possible Lorentz violations in the fermionic sector of the SME [7], with:

$$
\Gamma^{\mu}=\gamma^{\mu}+c^{\mu}{ }_{v} \gamma^{v}+d^{\mu}{ }_{v} \gamma^{v} \gamma^{5}, \quad M=m+b_{\mu} \gamma^{\mu} \gamma^{5}+\frac{H_{\mu v}}{2} \sigma^{\mu \nu}
$$

In particular, we will calculate the abelian axial anomaly for the choice $c^{\mu}{ }_{v}=0$ and $M=0$. We rewrite the Lagrangian (3) in a way that makes explicit the transformation 
properties of the indices involved. To this end, we follow the notation of the Dirac equation in curved space and write (3) as

$$
\mathcal{L}=\bar{\Psi}(x) i \gamma^{A} e_{A}^{\mu} \partial_{\mu} \Psi(x),
$$

where Latin indices $A=0,1,2,3:\{0, a=1,2,3\}$ live in the matrix space, while Greek indices $\mu=0,1,2,3:\{0, i=1,2,3\}$ label spacetime coordinates. Then, the indices $A$ transform under local Lorentz transformations while the indices $\mu$ transform under general coordinate transformations. In our flat space setting, both types of transformations coincide, but this distinction will be relevant when we make the continuation to Euclidean space.

We further constrain ourselves to the case:

$$
e_{A}^{\mu}=\delta_{A}^{\mu}+d^{\mu}{ }_{A} \gamma^{5},
$$

and we interpret $d^{\mu} A$ as four spacetime vectors labeled by the Dirac indices $A$. Moreover, the Lagrangian in Equation (3) yields the further restriction:

$$
d_{A}^{\mu}=\delta_{i}^{\mu} \delta_{A}^{0} v^{i},
$$

which says that we have only one spacetime vector $v_{0}^{\mu}$ corresponding to $A=0$ with only spatial components $v_{0}^{i}=v^{i}$. The main point here is that both types of indices have definite transformation properties when changing coordinate frames. In this notation, the gamma matrices satisfy $\left\{\gamma^{A}, \gamma^{B}\right\}=2 \eta^{A B}$ with $\eta^{A B}=\operatorname{diag}(+1,-1,-1,-1)$. The spacetime metric is $\eta^{\mu v}=\operatorname{diag}(+1,-1,-1,-1)$.

\section{A Brief on Weyl Semimetals}

In a low energy approximation near the Fermi energy, Weyl semimetals are materials for which their band structure can be described by a pair (or an even number) of nodes (Weyl points) separated in energy and momentum. The low energy theory of an isolated Weyl point is given by the Hamiltonian [16]:

$$
\mathrm{H}_{ \pm}(\mathbf{k})= \pm \sigma^{0} \mathbf{v}_{0} \cdot \mathbf{k}+\chi \sigma^{a} \mathbf{v}_{a} \cdot \mathbf{k}=\sigma^{A} v_{A}^{i}( \pm, \chi) k_{i}, \quad v_{A}^{i}( \pm, \chi)=\left( \pm v_{0}^{i}, \chi v_{a}^{i}\right),
$$

where $\sigma^{a}(a=1,2,3)$ are the Pauli matrices, $\sigma^{0}=I$ is the unit $2 \times 2$ matrix , $\chi= \pm 1$ is the chirality of the node and $\pm \mathbf{v}_{0}$ indicates the direction of the tilting. The Nielsen-Ninomiya theorem [14] states that each pair of cones must carry opposite chiralities. In Equation (9), $\mathbf{k}$ is the crystal momentum vector centered in each node. As shown in Ref. [21] for the untilted case, taking into account the separation of the nodes has direct consequences on the coefficient of the anomaly.

We have four spacetime vectors $\mathbf{v}_{A}=\left(v_{A}^{i}\right)(A=0,1,2,3, i=1,2,3)$ with only spatial components $v_{A}^{i}$. That is, for each particular label $i$, the object $v_{A}^{i}$ transforms as a covariant vector in Lorentz space. The vectors $v_{A}^{i}$ characterize the cone structure arising from the dispersion relations, where the apexes of the cones are located at the nodes. The vectors $\mathbf{v}_{a}$ describe the anisotropy of the cones while $\mathbf{v}_{0}$ gives their tilting. Our choice $\pm \mathbf{v}_{0}$ in the Hamiltonian (9) indicates that we are considering the two cones with tilting in the opposite directions.

The case of tilting in the same direction has been considered in Ref. [34] and results in no additional terms in the axial anomaly, yielding a Lorentz invariant result. Although the authors in Ref. [34] obtained a non-trivial anomaly in the case of cones with opposite tilting using the Fujikawa approach, their result differs from the one we found. Such difference arises from the alternative ways of dealing with the non-commuting operators involved. Since the calculation is very different from the standard case, it turns out that the correct expansion of the regulator required to define the anomaly is highly non-trivial and so it is worthy of the separate calculation which we perform. 
We will consider the isotropic case where $v_{a}^{j}=\delta_{a}^{j}$, such that the system to be studied only deals with the tilting of the cones and it is given by the Hamiltonian:

$$
\mathrm{H}=\mathrm{H}_{-} \oplus \mathrm{H}_{+}, \quad \mathrm{H}_{-}(\mathbf{k})=-\sigma^{0} \mathbf{v}_{0} \cdot \mathbf{k}-\boldsymbol{\sigma} \cdot \mathbf{k}, \quad \mathrm{H}_{+}(\mathbf{k})=+\sigma^{0} \mathbf{v}_{0} \cdot \mathbf{k}+\boldsymbol{\sigma} \cdot \mathbf{k} .
$$

Choosing the Weyl basis, we take:

$$
\gamma^{0}=\left(\begin{array}{ll}
0 & 1 \\
1 & 0
\end{array}\right), \quad \gamma^{a}=\left(\begin{array}{cc}
0 & \sigma^{a} \\
-\sigma^{a} & 0
\end{array}\right), \quad \gamma^{5}=\left(\begin{array}{cc}
-1 & 0 \\
0 & 1
\end{array}\right), \quad \alpha^{a}=\gamma^{0} \gamma^{a}=\left(\begin{array}{cc}
-\sigma^{a} & 0 \\
0 & \sigma^{a}
\end{array}\right) .
$$

in Minkowski space. Since the matrix $\gamma_{5}$ will play an important role in this work, we specify its definition together with our convention for the related Levi-Civita symbol $\epsilon_{M}^{\mu \nu \alpha \beta}$ :

$$
\gamma_{5}=i \gamma^{0} \gamma^{1} \gamma^{2} \gamma^{3}, \quad \operatorname{tr} \gamma_{5} \gamma^{\mu} \gamma^{v} \gamma^{\alpha} \gamma^{\beta}=-4 i \epsilon_{M}^{\mu \nu \alpha \beta}, \quad \epsilon_{M}^{0123}=+1 .
$$

On the basis of (11), the Hamiltonian $\mathrm{H}$ is:

$$
\mathrm{H}=\left(\begin{array}{cc}
\mathrm{H}_{+} & 0 \\
0 & \mathrm{H}_{-}
\end{array}\right)=\boldsymbol{\alpha} \cdot \mathbf{k}+\mathbf{v}_{0} \cdot \mathbf{k} \gamma^{5} .
$$

We then show that the Lagrangian of Equation (3) reproduces the Hamiltonian of Equation (13) before adding the electromagnetic coupling. We work in momentum space where $i D_{\mu} \longrightarrow i \partial_{\mu} \longrightarrow-k_{\mu}$. With this, a direct calculation from the Lagrangian (3) yields:

$$
\mathcal{L}=-\bar{\Psi}\left[\gamma^{A} k_{A}+\gamma^{0} \gamma^{5} \mathbf{v}_{0} \cdot \mathbf{k}\right] \Psi=-\Psi^{\dagger}\left[\left(k_{0}+\gamma^{5} \mathbf{v}^{0} \cdot \mathbf{k}\right)+\alpha^{a} k_{a}\right] \Psi,
$$

which gives $\mathrm{H}$ in Equation (13) after the Legendre transformation:

$$
\mathcal{H}=\Pi_{0}\left(\partial_{0} \psi\right)-\mathcal{L}=-\psi^{\dagger} k_{0} \psi-\mathcal{L}=\Psi^{\dagger} \mathrm{H} \Psi,
$$

is performed.

\section{The Axial Anomaly}

Before explaining the details, we give a quick overview on how the axial anomaly arises from a general chiral rotation in the fermionic functional for massless fermions, according to the general method of Fujikawa in the path integral approach [30,31]. The starting point is:

$$
\mathcal{Z}\left(A_{\mu}\right)=\int \mathcal{D} \bar{\Psi}^{\prime} \mathcal{D} \Psi^{\prime} \exp \left[i S\left(A_{\mu}, \Psi^{\prime}\right)\right]=\int \mathcal{D} \bar{\Psi}^{\prime} \mathcal{D} \Psi^{\prime} \exp \left[i \int d^{4} x\left(\bar{\Psi}^{\prime} i \mathbb{D} \Psi^{\prime}\right)\right],
$$

with $\mathbb{D}=\left(\gamma^{\mu} D_{\mu}+\gamma^{0} \gamma^{5} v^{i} D_{i}\right)=\Gamma^{\mu}\left(\partial_{\mu}-i e A_{\mu}\right)$. The change of integration variables $\Psi(x) \rightarrow \Psi^{\prime}(x)$ due to the chiral transformations:

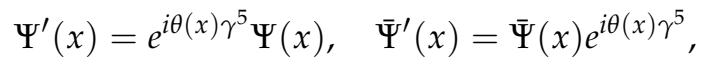

with $\theta(x)$ arbitrary, leaves the functional integral unchanged and produces:

$$
\mathcal{Z}\left(A_{\mu}\right)=\int J\left(A_{\mu}\right) \mathcal{D} \bar{\Psi} \mathcal{D} \Psi \exp \left[i \int d^{4} x\left(\bar{\Psi} i \mathbb{D} \Psi-J_{5}^{\mu} \partial_{\mu} \theta(x)\right)\right] .
$$

This allows the identification of the chiral current as $J_{5}^{\mu}=\bar{\Psi}(x) \Gamma^{\mu} \gamma_{5} \Psi(x)$ together with the introduction of the Jacobian $J\left(A_{\mu}\right)$ required by the change of variables $\mathcal{D} \Psi^{\prime} \mathcal{D} \Psi^{\prime}=$ $J\left(A_{\mu}\right) \mathcal{D} \bar{\Psi} \mathcal{D} \Psi$. The further calculation of $J\left(A_{\mu}\right)$ yields:

$$
J\left(A_{\mu}\right)=\exp \left(-i \int d^{4} x \theta(x) \mathcal{A}(x)\right) .
$$


Since $\delta \mathcal{Z}\left(A_{\mu}\right) / \delta \theta(x)=0$, it follows that:

$$
\partial_{\mu} J_{5}^{\mu}=\mathcal{A}(x),
$$

which defines the abelian chiral anomaly $\mathcal{A}(x)$ of the corresponding current.

The calculation is performed in Euclidean space and we follow the conventions of Ref. [30]. We perform a Wick rotation:

$$
x^{0} \longrightarrow-i x^{4}, \quad x_{0} \longrightarrow i x_{4}, \quad x_{4} \in \mathbb{R}, \quad \gamma^{0} \longrightarrow-i \gamma^{4},
$$

which applies to all tensorial indices. This leaves us with the Euclidean the metric $\eta_{\mu v}=$ $\operatorname{diag}(-1,-1,-1,-1)$ and the Lorentz indices $\mu$ now run from 1 to 4 . The Euclidean Dirac matrices satisfy:

$$
\left\{\gamma^{\mu}, \gamma^{\nu}\right\}=2 \eta^{\mu \nu}, \quad\left(\gamma^{\mu}\right)^{\dagger}=-\gamma^{\mu}
$$

and the pseudoscalar $\gamma^{5}$ retains its definition in Minkowski space:

$$
\gamma^{5}=i \gamma^{0} \gamma^{1} \gamma^{2} \gamma^{3}=\gamma^{4} \gamma^{1} \gamma^{2} \gamma^{3}=-\gamma^{1} \gamma^{2} \gamma^{3} \gamma^{4},
$$

with the basic properties:

$$
\operatorname{tr} \gamma_{5}=0, \quad \operatorname{tr} \gamma_{5} \gamma^{\mu} \gamma^{\nu}=0, \quad \operatorname{tr} \gamma_{5} \gamma^{\mu} \gamma^{\nu} \gamma^{\alpha} \gamma^{\beta}=-4 \epsilon_{E}^{\mu \nu \alpha \beta}, \quad \epsilon_{E}^{1234}=+1 .
$$

Since $v_{0}^{i}$ is contracted with the space indices of the covariant derivatives $D_{i}$, we consider these parameters as the space components of a vector which are not affected by the Wick rotation. Thus, we have:

$$
v_{0}^{i} \longrightarrow v_{4}^{i}, \quad v_{4}^{i} \in \mathbb{R} .
$$

With the above choices, the Euclidean Dirac operator:

$$
\mathbb{D}_{E}=\gamma^{\mu} D_{\mu}-i \gamma^{4} \gamma^{5} v_{4}^{k} D_{k}
$$

is Hermitian under the scalar product $\left(\psi, \mathbb{D}_{E} \chi\right)=\int d^{4} x \psi^{\dagger}(x) \mathbb{D}_{E} \chi(x)$. The Euclidean action is:

$$
S_{E}=\int d^{4} x \mathcal{L}_{E}, \quad \mathcal{L}_{E}=\bar{\Psi}(x) i \mathbb{D}_{E} \Psi(x),
$$

where $\bar{\Psi}(x)$ and $\Psi(x)$ are now independent fields. When going back to the Minkowski space, we set $\Psi \rightarrow \Psi_{M}, \bar{\Psi} \rightarrow\left(\Psi_{M}\right)^{\dagger} \gamma^{0}, d^{4} x \rightarrow i\left(d^{4} x\right)_{M}$ and $P^{\mu} \eta_{\mu v} Q^{v} \rightarrow\left(P^{\mu} \eta_{\mu v} Q^{v}\right)_{M}$, in addition to using the relations in Equation (21).

\subsection{The Modified Axial Current}

Our Lagrangian (27) is trivially invariant under the global transformations:

$$
\Psi(x) \longrightarrow e^{i \theta} \Psi(x), \quad \bar{\Psi}(x) \longrightarrow \bar{\Psi}(x) e^{-i \theta},
$$

which lead to the charge Noether current:

$$
J^{\mu}=\bar{\Psi} \gamma^{\mu} \Psi-i \bar{\Psi} \gamma^{4} \gamma^{5} v_{4}^{k} \delta_{k}^{\mu} \Psi,
$$

such that $\partial_{\mu} J^{\mu}=0$. The invariance of the Lagrangian can be extended to transformations including a local parameter $\theta(x)$, provided we demand that $A_{\mu} \longrightarrow A_{\mu}-\partial_{\mu} \theta(x) / e$. Now, the conservation of $J^{\mu}$ is a consequence of gauge invariance, and must still hold in the quantum version of the theory. In other words, no anomalies are acceptable for this current.

The massless classical Lagrangian (27) has an additional symmetry corresponding to the chiral transformations (17) with the constant parameter $\theta$. The associated Noether cur- 
rent, which is conserved due to the classical equations of motion, can be readily identified by an arbitrary infinitesimal variation of the fields:

$$
\delta \bar{\Psi}(x)=i \delta \theta(x) \bar{\Psi} \gamma^{5}, \quad \delta \Psi(x)=i \delta \theta(x) \gamma^{5} \Psi(x)
$$

resulting when promoting $\delta \theta$ to a local parameter. The equations of motions yield:

$$
0=\delta S_{E}=\delta \int d^{4} x \delta \mathcal{L}_{E}=-\int d^{4} x\left(\partial_{\mu} \delta \theta\right)\left[\bar{\Psi} \gamma^{\mu} \gamma^{5} \Psi-i\left(v_{4}^{k} \delta_{k}^{\mu}\right) \bar{\Psi} \gamma^{4} \Psi\right],
$$

from where we read the conservation of the modified classical axial current:

$$
J_{5}^{\mu}=\bar{\Psi} \gamma^{\mu} \gamma^{5} \Psi-i\left(v_{4}^{k} \delta_{k}^{\mu}\right) \bar{\Psi} \gamma^{4} \Psi,
$$

since $\delta \theta(x)$ is arbitrary. In quantum electrodynamics, it is not possible to maintain the conservation of both $J^{\mu}$ and $J_{5}^{\mu}$, and one must choose the conservation of the electric charge to preserve gauge invariance. This leads to the presence of the chiral anomaly.

\subsection{The Fujikawa Method}

As we previously mentioned, the path integral calculation of the chiral anomaly [30] tells us that its origin is found in the non-trivial Jacobian, arising when performing the axial transformation. Using this method, we will compute the axial anomaly originating from our modified Lagrangian (27). We start from the Euclidean path integral:

$$
\mathcal{Z}_{E}\left(A_{\mu}\right)=\int \mathcal{D} \bar{\Psi}_{E} \mathcal{D} \Psi_{E} \exp S_{E}=\int \mathcal{D} \bar{\Psi}_{E} \mathcal{D} \Psi_{E} \exp \left[\int\left(d^{4} x\right)_{E}\left(\bar{\Psi}_{E} i \mathbb{D}_{E} \Psi_{E}\right)\right],
$$

where we suppress the subindices $E$ in the following. Recalling that our modified Dirac operator $\mathbb{D}$ is Hermitian, with eigenfunctions $\varphi_{n}(x)$ such that $\mathbb{D} \varphi_{n}=\lambda_{n} \varphi_{n}$, we follow the standard steps in the calculation of the Jacobian by expanding the fields in terms of the orthonormal and complete eigenvectors $\varphi_{n}(x)$. That is:

$$
\Psi(x)=\sum_{n} a_{n} \varphi_{n}(x), \quad \bar{\Psi}(x)=\sum_{n} \bar{b}_{n} \varphi_{n}^{\dagger}(x),
$$

and similarly for $\bar{\Psi}^{\prime}$ and $\Psi^{\prime}$. Let us recall that the fields are Grassmann numbers and so are the coefficients $a_{n}, a_{n}^{\prime}$ and $\bar{b}_{n}, \bar{b}_{n}^{\prime}$. The infinitesimal axial transformation (30) yields the relation:

$$
a_{n}^{\prime}=\left(\delta_{n, m}+i \int \mathrm{d}^{4} x \varphi_{n}^{\dagger}(x) \delta \theta(x) \gamma^{5} \varphi_{m}(x)\right) a_{n} \equiv T_{m n} a_{n},
$$

with a similar expression for $\bar{b}_{n}^{\prime}$ in terms of $\bar{b}_{n}$. The above equation introduces the matrix $T=I+\delta t$ with:

$$
\delta t_{m n}=i \int \mathrm{d}^{4} x \varphi_{n}^{\dagger}(x) \delta \theta(x) \gamma^{5} \varphi_{m}(x) .
$$

The Grassmann character of the expansion coefficients leads to:

$$
\mathcal{D} \bar{\Psi}^{\prime} \mathcal{D} \Psi^{\prime}=\lim _{N \longrightarrow \infty} \prod_{n=1}^{N} \mathrm{~d} \bar{b}_{n}^{\prime} \mathrm{d} a_{n}^{\prime}=(\operatorname{det} T)^{-2} \lim _{N \longrightarrow \infty} \prod_{n=1}^{N} \mathrm{~d} \bar{b}_{n} \mathrm{~d} a_{n}=(\operatorname{det} T)^{-2} \mathcal{D} \bar{\Psi} \mathcal{D} \Psi,
$$

which identifies $J=(\operatorname{det} T)^{-2}$ as the Jacobian of the transformation. As $\operatorname{det}(M)=$ $\exp [\operatorname{Tr}(\ln (M))]$, we have:

$$
\begin{aligned}
& J_{\delta \theta}=(\operatorname{det} T)^{-2}=\exp [-2 \operatorname{Tr}(\ln (I+\delta t))]=\exp [-2 \operatorname{Tr}(\delta t)], \\
& J_{\delta \theta}=\exp \left[-2 i \sum_{n} \int \mathrm{d}^{4} x \varphi_{n}^{\dagger}(x) \delta \theta(x) \gamma^{5} \varphi_{n}(x)\right]=\exp \left[-2 i \operatorname{Tr}\left(\delta \theta \gamma^{5}\right)\right],
\end{aligned}
$$


to the first order in $\delta \theta(x)$. Here, the trace $\operatorname{Tr}$ is taken in the matrix space $\left(\sum_{n}\right)$ as well as in the coordinate space $\left(\int \mathrm{d}^{4} x\right)$. Nevertheless, the series in the exponential diverges, and so it must be regularized. We need to preserve invariance under the gauge transformations:

$$
\varphi_{n}^{\prime}(x)=e^{i \Lambda(x)} \varphi_{n}(x), \quad A_{\mu}^{\prime}=A_{\mu}-\frac{1}{e} \partial_{\mu} \Lambda(x)
$$

which induce the transformation $\mathbb{D} e^{i \Lambda(x)}=e^{i \Lambda(x)} \mathbb{D}$ such that $\varphi_{n}^{\dagger}(x) F(\mathbb{D}) \varphi_{n}(x)$ is gauge invariant. To ensure convergence, we choose $F(\mathbb{D})=\exp \left[-\mathbb{D}^{2} / M^{2}\right]$ with $M \rightarrow \infty$ at the end of the calculation.

It is convenient to define:

$$
\alpha(x) \equiv \lim _{M \longrightarrow \infty} \sum_{n} \varphi_{n}^{\dagger}(x) \gamma^{5} e^{-\mathbb{D}^{2} / M^{2}} \varphi_{n}(x),
$$

such that the regularized Jacobian is written as

$$
J\left(A_{\mu}\right)=\exp \left[-2 i \int \mathrm{d}^{4} x \delta \theta(x) \alpha(x)\right] .
$$

This expression for the regularized Jacobian, together with the change in the Euclidean action (33) due to the chiral transformations (30), enters in the functional integral by modifying the Euclidean Lagrangian (27) as

$$
\mathcal{L}_{E} \rightarrow \mathcal{L}_{E}+\delta \theta(x)\left(\partial_{\mu} J_{5}^{\mu}-2 i \alpha(x)\right)
$$

where $\alpha(x)$ depends on the external electromagnetic field and $J_{5}^{\mu}$ is given by Equation (32). Let us emphasize that to calculate the chiral anomaly, it is sufficient to consider only the infinitesimal transformations (30).

Having regularized the Jacobian, we take a convenient change of basis, from $\left\{\varphi_{n}(x)\right\}$ to $\left\{e^{i k \cdot x}\right\}$, so that:

$$
\alpha(x)=\lim _{M \longrightarrow \infty} \operatorname{tr} \int \frac{\mathrm{d}^{4} k}{(2 \pi)^{4}} e^{-i k \cdot x} \gamma^{5} e^{-\mathbb{D}^{2} / M^{2}} e^{i k \cdot x} .
$$

Here, tr refers to the trace in the gamma matrix space. We then calculate $\mathbb{D}^{2}$ and organize the resulting terms to evaluate $\alpha(x)$. Using the relation $\left[D_{\mu}, D_{v}\right]=-i e F_{\mu v}$, we obtain:

$$
\mathbb{D}^{2}=D_{\mu} D^{\mu}-\frac{i e}{4}\left[\gamma^{\mu}, \gamma^{v}\right] F_{\mu \nu}-e \gamma^{\mu} \gamma^{4} \gamma^{5} v_{4}^{j} F_{\mu j}-2 i \gamma^{a} \gamma^{4} \gamma^{5} v_{4}^{j} D_{j} D_{a}-\left(v_{4}^{i} D_{i}\right)^{2} .
$$

As $\left[D_{\mu}, e^{i k \cdot x}\right]=i k_{\mu} e^{i k \cdot x}$, we can remove the exponentials in Equation (43) by shifting $D_{\mu} \rightarrow D_{\mu}+i k_{\mu}$. This leads to:

$$
\begin{aligned}
\alpha(x)=\lim _{M \longrightarrow \infty} \operatorname{tr} \gamma^{5} \int \frac{\mathrm{d}^{4} k}{(2 \pi)^{4}} & \exp \frac{-1}{M^{2}}\left[\left(i k_{\mu}+D_{\mu}\right)^{2}-\frac{i e}{4}\left[\gamma^{\mu}, \gamma^{v}\right] F_{\mu v}-e \gamma^{\mu} \gamma^{4} \gamma^{5} v_{4}^{i} F_{\mu i}\right. \\
& \left.-2 i \gamma^{i} \gamma^{4} \gamma^{5} v_{4}^{j}\left(i k_{j}+D_{j}\right)\left(i k_{i}+D_{i}\right)-\left(v_{4}^{j}\left(i k_{j}+D_{j}\right)\right)^{2}\right] .
\end{aligned}
$$

Scaling the momentum integral $k_{\mu} \longrightarrow M k_{\mu}$, and defining $\lambda=1 / M$, we obtain the final expression:

$$
\alpha(x)=\lim _{\lambda \longrightarrow 0} \int \frac{\mathrm{d}^{4} k}{(2 \pi)^{4}} e^{-k_{\mu} k_{\mu}-\left(v_{a}^{4} k_{a}\right)^{2}}\left(\frac{1}{\lambda^{4}} \operatorname{tr} \gamma^{5} e^{\mathbb{A}+\lambda \mathbb{B}}\right) .
$$


with the identifications:

$$
\mathbb{A}=-2 i \gamma^{i} \gamma^{4} \gamma^{5} v_{4}^{j} k_{j} k_{i}, \quad \mathbb{B}=\alpha+\beta_{i} \gamma^{i} \gamma^{4} \gamma^{5}+\frac{i e}{2} \lambda\left(\gamma^{\mu} \gamma^{v} F_{\mu \nu}-2 i \gamma^{\mu} \gamma^{4} \gamma^{5} v_{4}^{j} F_{\mu j}\right),
$$

and the further splitting:

$$
\alpha=2 i\left(v_{4}^{i} k_{i} v_{4}^{j} D_{j}-k_{\mu} D^{\mu}\right)+\lambda\left(\left(v_{4}^{j} D_{j}\right)^{2}-D_{\mu} D^{\mu}\right), \quad \beta_{i}=-2 v_{4}^{j}\left(k_{j} D_{i}+k_{i} D_{j}\right)+2 i \lambda v_{4}^{j} D_{j} D_{i} .
$$

As the operators $\mathbb{A}$ and $\mathbb{B}$ do not commute, the factorization $e^{\mathbb{A}+\lambda \mathbb{B}}=e^{\mathbb{A}} e^{\lambda \mathbb{B}}$ followed by an expansion of $e^{\lambda \mathbb{B}}$ in a Taylor series of $\lambda \mathbb{B}$ is not allowed. We must therefore take the complete expansion:

$$
e^{\mathbb{A}+\lambda \mathbb{B}}=\sum_{n=0}^{\infty} \frac{1}{n !}(\mathbb{A}+\lambda \mathbb{B})^{n} .
$$

This includes terms to all orders of $v_{4}^{i}$, even when focusing on those proportional to $\lambda^{4}$, which are the only ones which contribute to $\alpha(x)$.

In this work, we restrict ourselves to calculate the contributions to $\alpha(x)$ only to the first and second order in $v_{4}^{i}$. This means that we are considering $v_{4}^{i}$ as a small parameter, which limits the range of our results in the context of further applications to Weyl semimetals. With this assumption, we need to identify the required powers of $v_{4}^{i}$ in $\mathbb{A}$ and $\mathbb{B}$. To simplify the notation, we write $v_{4}^{i}=v^{i}$ in what follows:

$$
\begin{aligned}
& \mathbb{A}=v^{j} M_{j}, \quad M_{j}=-2 i\left(\gamma^{i} \gamma^{4} \gamma^{5}\right) k_{j} k_{i}, \\
& \mathbb{B}=N_{0}+v^{j} N_{j}+v^{i} v^{j} N_{i j}, \\
& N_{0}=-2 i k_{\mu} D^{\mu}+\lambda\left(\frac{i e}{2} \gamma^{\mu} \gamma^{v} F_{\mu v}-D_{\mu} D^{\mu}\right), \\
& N_{j}=-2\left(k_{j} D_{i}+k_{i} D_{j}\right) \gamma^{i} \gamma^{4} \gamma^{5}+\lambda\left(2 i D_{j} D_{i} \gamma^{i} \gamma^{4} \gamma^{5}+e \gamma^{\mu} \gamma^{4} \gamma^{5} F_{\mu j}\right), \\
& N_{i j}=2 i k_{i} D_{j}+\lambda D_{i} D_{j} .
\end{aligned}
$$

Since any contribution to $\alpha(x)$ arises from the term proportional to $\lambda^{4}$ in the expansion of the exponential (49), we still have to explicitly write the $\lambda$-dependence of the above coefficients. This yields the additional splitting:

$$
N_{0}=N_{00}+\lambda N_{01}, \quad N_{j}=N_{j 0}+\lambda N_{j 1}, \quad N_{i j}=N_{i j 0}+\lambda N_{i j 1},
$$

with:

$$
\begin{aligned}
N_{00} & =-2 i k_{\mu} D^{\mu}, \quad N_{01}=\left(\frac{i e}{2} \gamma^{\mu} \gamma^{v} F_{\mu \nu}-D_{\mu} D^{\mu}\right), \\
N_{j 0} & =-2\left(k_{j} D_{i}+k_{i} D_{j}\right)\left(\gamma^{i} \gamma^{4} \gamma^{5}\right), \\
N_{j 1} & =\left[e \gamma^{\mu} \gamma^{4} \gamma^{5} F_{\mu j}+2 i D_{j} D_{i}\left(\gamma^{i} \gamma^{4} \gamma^{5}\right)\right], \\
N_{i j 0} & =2 i k_{i} D_{j}, \quad N_{i j 1}=D_{i} D_{j} .
\end{aligned}
$$

Let us observe that all the operators in the previous four equations commute with $\gamma_{5}$. Since the binomial expansion in Equation (49) will produce multiple permutations of the different coordinate operators $\left(D_{\mu}\right.$ and $\left.F_{\mu v}\right)$, it would be tempting to use the cyclic property of the trace in coordinate space to move operators from the far right to the far left of some expressions and therefore simplify the proliferation of non-commutative contributions. Nevertheless, this would not be useful in the case of derivatives because of the term $\delta \theta(x)$ inside the spacetime trace. Suppose we have a term like $\operatorname{Tr} \delta \theta(x) \gamma_{5} D_{\mu} F_{\alpha \beta} D_{v}$, then the cyclic identity will yield $\operatorname{Tr} D_{v} \delta \theta(x) \gamma_{5} D_{\mu} F_{\alpha \beta}$ which is different from $\operatorname{Tr} \delta \theta(x) \gamma_{5} D_{v} D_{\mu} F_{\alpha \beta}$ since 
$\left[D_{v}, \delta \theta(x)\right]=-\left(\partial_{v} \delta \theta(x)\right) \neq 0$. For this reason, we only use the cyclic identity of the trace tr in matrix space in our calculations.

\section{The First Order Expansion}

We look for the first order contribution in $v^{i}$ to the anomaly $\alpha(x)$ defined in Equation (46). To take into account the non-commuting operators that are involved, we start by rewriting the term $(\mathbb{A}+\lambda \mathbb{B})^{n}$ to be expanded so that the dependence upon $v_{a}$ is made explicit:

$$
\begin{aligned}
& \frac{1}{n !}(\mathbb{A}+\lambda \mathbb{B})^{n}=\frac{1}{n !}\left(A+B_{i} v^{i}+v^{i} v^{j} C_{i j}\right)^{n}, \\
& A=\lambda N_{0}, \quad B_{i}=M_{i}+\lambda N_{i}, \quad C_{i j}=\lambda N_{i j} .
\end{aligned}
$$

The coefficients $N_{0}, M_{i}, N_{i}, N_{i j}$ were previously defined in Equations (50) and (53). As previously emphasized, we still have to make explicit the $\lambda$-dependence of the expansion and only focus on the $\lambda^{4}$ contribution. Furthermore, we recall that $\gamma^{5}$ commutes with all the remaining operators. The contribution to each order $n$ is denoted by $a_{n}$. In this way, when looking for the linear contribution in $v^{i}$, it is sufficient to consider the terms:

$$
\frac{1}{n !}(\mathbb{A}+\lambda \mathbb{B})^{n} \rightarrow \frac{1}{n !}\left\lfloor A^{n-1} B_{i}\right\rfloor v^{i} \equiv a_{n}
$$

where $\left\lfloor A^{n-1} B_{i}\right\rfloor$ is a short-hand notation for all possible ordering arising in a given product of operators which do not commute. For example, in this case, the coefficients $a_{n}$ are:

$$
\begin{aligned}
& a_{2}=\frac{1}{2 !}\left\lfloor A, B_{i}\right\rfloor v^{i}=\frac{1}{2 !}\left(A B_{i}+B_{i} A\right) v^{i}, \\
& a_{3}=\frac{1}{3 !}\left\lfloor A^{2}, B_{i}\right\rfloor v^{i}=\frac{1}{3 !}\left(A B_{i} A+B_{i} A^{2}+A^{2} B_{i}\right) v^{i}, \\
& a_{4}=\frac{1}{4 !}\left\lfloor A^{3}, B_{i}\right\rfloor v^{i}=\frac{1}{4 !}\left(A B_{i} A^{2}+B_{i} A^{3}+A^{2} B_{i} A+A^{3} B_{i}\right) v^{i}, \\
& a_{5}=\frac{1}{5 !}\left\lfloor A^{4}, B_{i}\right\rfloor v^{i}=\frac{1}{5 !}\left(A^{4} B_{i}+A B_{i} A^{3}+A^{2} B_{i} A^{2}+A^{3} B_{i} A+B_{i} A^{4}\right) v^{i} .
\end{aligned}
$$

Since $A=\lambda N_{0}$, all contributions $a_{n}$ with $n \geq 6$ will yield a zero result.

Now, we make explicit the $\lambda$-dependence in order to extract the $\lambda^{4}$ terms. To this end, we use Equation (58) together with the further expansion in powers of $\lambda$ given in Equation (53). This yields:

$$
\begin{aligned}
& a_{2}=\frac{1}{2 !} \lambda\left\lfloor\left(N_{00}+\lambda N_{01}\right),\left(M_{i}+\lambda N_{i 0}+\lambda^{2} N_{i 1}\right)\right\rfloor_{2} v^{i}, \\
& a_{3}=\frac{1}{3 !}\left\lfloor\lambda^{2}\left(N_{00}+\lambda N_{01}\right)^{2},\left(M_{i}+\lambda N_{i 0}+\lambda^{2} N_{i 1}\right)\right\rfloor_{3} v^{i}, \\
& a_{4}=\frac{1}{4 !}\left\lfloor\lambda^{3}\left(N_{00}+\lambda N_{01}\right)^{3},\left(M_{i}+\lambda N_{i 0}+\lambda^{2} N_{i 1}\right)\right\rfloor_{4} v^{i}, \\
& a_{5}=\frac{1}{5 !}\left\lfloor\lambda^{4}\left(N_{00}+\lambda N_{01}\right)^{4},\left(M_{i}+\lambda N_{i 0}+\lambda^{2} N_{i 1}\right)\right\rfloor_{5} v^{i},
\end{aligned}
$$

from where we extract the coefficients of $\lambda^{4}$. The result is:

$$
\begin{aligned}
& \frac{a_{2}}{\lambda^{4}}=\frac{1}{2 !}\left\lfloor N_{01}, N_{i 1}\right\rfloor_{2} v^{i}, \\
& \frac{a_{3}}{\lambda^{4}}=\frac{1}{3 !}\left[\left\lfloor N_{01}^{2}, M_{i}\right\rfloor_{3}+\left\lfloor N_{00}, N_{01}, N_{i 0}\right\rfloor_{6}+\left\lfloor N_{00}^{2}, N_{i 1}\right\rfloor_{3}\right\rfloor v^{i}, \\
& \frac{a_{4}}{\lambda^{4}}=\frac{1}{4 !}\left[\left\lfloor N_{00}^{2}, N_{01}, M_{i}\right\rfloor_{24}+\left\lfloor N_{00}^{3}, N_{i 0}\right\rfloor_{4}\right\rfloor v^{i}, \\
& \frac{a_{5}}{\lambda^{4}}=\frac{1}{5 !}\left\lfloor N_{00}^{4}, M_{i}\right\rfloor_{5} v^{i} .
\end{aligned}
$$


The notation is $\lfloor P, Q, \ldots R\rfloor_{n}$, where $n$ denotes the total number of non-commuting products inside the bracket $\lfloor\ldots\rfloor$. Now, we take the trace in matrix space including $\gamma^{5}$, obtaining:

$$
\begin{aligned}
& \operatorname{tr}\left(\gamma^{5}\left\lfloor N_{01}, N_{j 1}\right\rfloor_{2}\right)=8 i e^{2} F^{4 \mu} F_{\mu j}+4 e\left\{F_{4 j}, D_{\mu} D^{\mu}\right\}-8 e\left\{D_{j} D_{i}, F^{4 i}\right\}, \\
& \operatorname{tr}\left(\gamma^{5}\left\lfloor N_{01}^{2}, M_{j}\right\rfloor_{3}\right)=24 e\left(k_{j} k_{i}\right)\left\{D_{\alpha} D^{\alpha}, F_{4 i}\right\}, \\
& \operatorname{tr}\left(\gamma_{5}\left\lfloor N_{00}^{2}, N_{j 1}\right\rfloor_{3}\right)=16 e\left[\left\{\left(k_{\mu} D^{\mu}\right)^{2}, F_{4 j}\right\}+\left(k_{\mu} D^{\mu}\right) F_{4 j}\left(k_{\mu} D^{\mu}\right)\right], \\
& \operatorname{tr}\left(\gamma^{5}\left\lfloor N_{00}, N_{01}, N_{j 0}\right\rfloor_{6}\right)=-16 e\left(k_{\alpha} D^{\alpha}\right)\left\{F_{4 m},\left(k_{j} D_{m}+k_{m} D_{j}\right)\right\} \\
&-16 e\left(k_{j} D_{m}+k_{m} D_{j}\right)\left\{k_{\alpha} D^{\alpha}, F_{4 m}\right\} \\
&-16 e F_{4 m}\left\{k_{\alpha} D^{\alpha},\left(k_{j} D_{m}+k_{m} D_{j}\right)\right\}, \\
& \operatorname{tr}\left(\gamma^{5}\left\lfloor N_{00}^{2}, N_{01}, M_{j}\right\rfloor_{24}\right)=-128 e k_{j} k_{i}\left[\left\{\left(k_{\mu} D^{\mu}\right)^{2}, F_{4 i}\right\}+\left(k_{\mu} D^{\mu}\right) F_{4 i}\left(k_{\alpha} D^{\alpha}\right)\right], \\
& \operatorname{tr}\left(\gamma^{5}\left\lfloor N_{00}^{3}, N_{j 0}\right\rfloor_{4}\right)=0, \quad \operatorname{tr}\left(\gamma^{5}\left\lfloor N_{00}^{4}, M_{j}\right\rfloor_{4}\right)=0 .
\end{aligned}
$$

In the above equations, we factored out the term $v^{j}$ and $\{P, Q\}=P Q+Q P$ denotes the anticommutator of $A$ and $B$.

We then perform the integrals with respect to the momentum using the relations:

$$
\begin{aligned}
& \int d^{4} k e^{-k_{\mu} k_{\mu}}() \rightarrow \pi^{2}(), \quad \int d^{4} k e^{-k_{\mu} k_{\mu}} k_{\alpha} k_{\beta}()=\frac{\pi^{2}}{2} \delta_{\alpha \beta}(), \\
& \int d^{4} k e^{-k_{\mu} k_{\mu}} k_{\alpha} k_{\beta} k_{\mu} k_{v}()=\frac{\pi^{2}}{4}\left(\delta_{\alpha \beta} \delta_{\mu v}+\delta_{\alpha \mu} \delta_{\beta v}+\delta_{\alpha v} \delta_{\beta \mu}\right)() .
\end{aligned}
$$

Going back to the notation in Equations (65)-(68), we obtain:

$$
\begin{aligned}
\frac{1}{\pi^{2} \lambda^{4}} \int d^{4} k e^{-k_{\mu} k_{\mu}} \operatorname{tr}\left(\gamma^{5} a_{2}\right)_{j} & =4 i e^{2} F_{4 \mu} F_{\mu j}+2 e\left\{D^{2}, F_{4 j}\right\}-4 e\left\{F_{4 i}, D_{j} D_{i}\right\} \\
\frac{1}{\pi^{2} \lambda^{4}} \int d^{4} k e^{-k_{\mu} k_{\mu}} \operatorname{tr}\left(\gamma^{5} a_{3}\right)_{j}= & -2 e\left\{D^{2}, F_{4 j}\right\}-\frac{4}{3} e\left[\left\{D^{2}, F_{4 j}\right\}+D^{\mu} F_{4 j} D_{\mu}\right] \\
& +\frac{8}{3} e\left(D_{j} F^{4 i} D_{i}+D_{i} F^{4 i} D_{j}+\left\{F^{4 i},\left\{D_{j}, D_{i}\right\}\right\}\right), \\
\frac{1}{\pi^{2} \lambda^{4}} \int d^{4} k e^{-k_{\mu} k_{\mu}} \operatorname{tr}\left(\gamma^{5} a_{4}\right)_{j}= & +\frac{4}{3} e\left(\left\{D^{2}, F_{4 j}\right\}+D^{\mu} F_{4 j} D_{\mu}\right) \\
& -\frac{4}{3} e\left(\left\{\left\{D_{j}, D_{i}\right\}, F_{4 i}\right\}+D_{j} F_{4 i} D_{i}+D_{i} F_{4 i} D_{j}\right) .
\end{aligned}
$$

Here, $D^{2}=D_{\mu} D^{\mu}$. In this notation, we write the contribution $\alpha^{(1)}(x)$ to the anomaly, which is linear in $v^{j}$, as

$$
(4 \pi)^{2} \alpha^{(1)}(x)=\frac{1}{\pi^{2} \lambda^{4}} \int d^{4} k e^{-k_{\mu} k_{\mu}} \operatorname{tr}\left(\gamma_{5} a_{j}\right) v^{j}, \quad a_{j}=\left(a_{2}+a_{3}+a_{4}\right)_{j}
$$

The direct combination of the previous equations in (79) cancels the $D^{2}$ terms and provides commutators of covariant derivatives which can be traded by the corresponding field strength. We are left with:

$$
(4 \pi)^{2} \alpha^{(1)}(x)=-i \frac{4}{3} e^{2} F_{4 i} F_{i j} v^{j}+\frac{4}{3} e\left(D_{i} F_{4 i} D_{j}-F^{4 i} D_{i} D_{j}+D_{j} F_{4 i} D_{i}-D_{i} D_{j} F_{4 i}\right) v^{j} .
$$


In order to show the gauge invariance of our result, we rearrange the terms with covariant derivatives in the following way:

$$
\begin{gathered}
D_{i} F_{4 i} D_{j}-F_{4 i} D_{i} D_{j}=D_{i} F_{4 i} D_{j}-\left(\left[F_{4 i}, D_{i}\right]+D_{i} F_{4 i}\right) D_{j}=\left(\partial_{i} F^{4 i}\right) D_{j}, \\
D_{j} F_{4 i} D_{i}-D_{i} D_{j} F_{4 i}=D_{j}\left[F_{4 i}, D_{i}\right]+\left(D_{j} D_{i}-D_{i} D_{j}\right) F_{4 i}=-D_{j}\left(\partial_{i} F_{4 i}\right)-i e F_{j i} F_{4 i} .
\end{gathered}
$$

Substituting in Equation (80) yields:

$$
\begin{aligned}
(4 \pi)^{2} \alpha^{(1)}(x)= & -i \frac{4}{3} e^{2} F_{4 i} F_{i j} v^{j}+\frac{4}{3} e\left(\left(\partial_{i} F^{4 i}\right) D_{j}-D_{j}\left(\partial_{i} F_{4 i}\right)-i e F_{j i} F_{4 i}\right) v^{j} \\
= & \frac{4}{3} e v^{j}\left(\left(\partial_{i} F^{4 i}\right) D_{j}-D_{j}\left(\partial_{i} F_{4 i}\right)\right) \\
= & \frac{4}{3} e v^{j}\left(\left(\partial_{i} F^{4 i}\right)\left(-i e A_{j}\right)-\left(\partial_{j} \partial_{i} F_{4 i}\right)+\left(i e A_{j}\right)\left(\partial_{i} F_{4 i}\right)\right)=-\frac{4}{3} e v^{j} \partial_{j} \partial_{i} F_{4 i} .
\end{aligned}
$$

Nevertheless, we can discard this unexpected gauge invariant term, recalling that a redefinition of the current is allowed provided the value of the charge remains unchanged. In our case, we show that the contribution of $\alpha^{(1)}(x)$ to the chiral charge is zero. Returning to the Minkowski space, the additional term in the divergence of the axial current is $\partial^{\mu} J_{5}^{(1) \mu} \sim e v^{j} \partial_{j} \partial_{\mu} F^{\mu 0}=\partial_{\mu}\left(e v^{j} \partial_{j} F^{\mu 0}\right)$, which yields the extra term $J_{5}^{(1) \mu}=e v^{j} \partial_{j} F^{\mu 0}$ in the axial current. The corresponding charge:

$$
Q_{5}{ }^{(1)}=\int d^{3} x J_{5}{ }^{(1) 0}
$$

is identically zero because $F^{00}=0$. Thus, we find a null contribution to the anomaly at first order in $v^{i}$.

\section{The Second Order Expansion}

Since in flat space there is no difference among spacetime and Lorentz indices, we include $a, b, \ldots$ among the space indices in the following. Having obtained that there are no linear contributions of $v^{a}$ to the anomaly, we now focus on the quadratic ones. They are included in:

$$
\begin{aligned}
\frac{1}{n !}(\mathbb{A}+\lambda \mathbb{B})^{n} \rightarrow & \frac{1}{n !}\left\lfloor\left(\lambda N_{0}\right)^{n-2},\left(M_{a}+\lambda N_{a}\right),\left(M_{b}+\lambda N_{b}\right)\right\rfloor_{\left(\begin{array}{c}
n \\
2
\end{array}\right)} v^{a} v^{b} \\
& +\frac{1}{n !}\left\lfloor\left(\lambda N_{0}\right)^{n-1},\left(\lambda N_{a b}\right)\right\rfloor_{n} v^{a} v^{b} \\
& \equiv b_{n}+c_{n},
\end{aligned}
$$

where $b_{n}$ and $c_{n}$ denote the first and second term in the right-hand side of Equation (85), respectively. Let us also keep in mind the definition of the symbol $\lfloor\ldots\rfloor$ given in Equation (60).

The only relevant contributions ( $\lambda$ of order less than 5 ) are $b_{2}, b_{3}, b_{4}, b_{5}, b_{6}$ and $c_{1}, c_{2}$, $c_{3}, c_{4}$. This means:

$$
\begin{aligned}
& b_{2}=\frac{1}{2}\left\lfloor\left(M_{a}+\lambda N_{a}\right)\left(M_{b}+\lambda N_{b}\right)\right\rfloor_{1} v^{a} v^{b}, \\
& b_{3}=\frac{1}{6}\left\lfloor\left(\lambda N_{0}\right),\left(M_{a}+\lambda N_{a}\right)\left(M_{b}+\lambda N_{b}\right)\right\rfloor_{3} v^{a} v^{b}, \\
& b_{4}=\frac{1}{24}\left\lfloor\left(\lambda N_{0}\right)^{2},\left(M_{a}+\lambda N_{a}\right)\left(M_{b}+\lambda N_{b}\right)\right\rfloor_{6} v^{a} v^{b}, \\
& b_{5}=\frac{1}{120}\left\lfloor\left(\lambda N_{0}\right)^{3},\left(M_{a}+\lambda N_{a}\right)\left(M_{b}+\lambda N_{b}\right)\right\rfloor_{10} v^{a} v^{b}, \\
& b_{6}=\frac{1}{720}\left\lfloor\left(\lambda N_{0}\right)^{4},\left(M_{a}+\lambda N_{a}\right)\left(M_{b}+\lambda N_{b}\right)\right\rfloor_{15} v^{a} v^{b},
\end{aligned}
$$


and:

$$
\begin{aligned}
& c_{1}=\left\lfloor\lambda N_{a b}\right\rfloor_{1} v^{a} v^{b}, \quad c_{2}=\frac{1}{2}\left\lfloor\left(\lambda N_{0}\right),\left(\lambda N_{a b}\right)\right\rfloor_{2} v^{a} v^{b}, \\
& c_{3}=\frac{1}{6}\left\lfloor\left(\lambda N_{0}\right)^{2},\left(\lambda N_{a b}\right)\right\rfloor_{3} v^{a} v^{b}, \quad c_{4}=\frac{1}{24}\left\lfloor\left(\lambda N_{0}\right)^{3},\left(\lambda N_{a b}\right)\right\rfloor_{4} v^{a} v^{b} .
\end{aligned}
$$

In our notation, no permutations are made between the operators $\left(M_{a}+\lambda N_{a}\right)$ and $\left(M_{b}+\lambda N_{b}\right)$ as they are the same when contracted with $v_{a}$ and $v_{b}$. To emphasize this, we introduced the further convention that only the terms separated with commas in $\lfloor\ldots\rfloor$ are subjected to permutations. For clarity, the subindex in $\lfloor\ldots\rfloor$ indicates the number of permutations in the bracket, which is given by a multinomial coefficient. Making explicit the full dependence in $\lambda$, we have:

$$
\begin{aligned}
& b_{2}=\frac{1}{2}\left\lfloor\left(M_{a}+\lambda N_{a 0}+\lambda^{2} N_{a 1}\right)\left(M_{b}+\lambda N_{b 0}+\lambda^{2} N_{b 1}\right)\right\rfloor_{1} v^{a} v^{b}, \\
& b_{3}=\frac{1}{6}\left\lfloor\lambda\left(N_{00}+\lambda N_{01}\right),\left(M_{a}+\lambda N_{a 0}+\lambda^{2} N_{a 1}\right)\left(M_{b}+\lambda N_{b 0}+\lambda^{2} N_{b 1}\right)\right\rfloor_{3} v^{a} v^{b}, \\
& b_{4}=\frac{1}{24}\left\lfloor\lambda^{2}\left(N_{00}+\lambda N_{01}\right)^{2},\left(M_{a}+\lambda N_{a 0}+\lambda^{2} N_{a 1}\right)\left(M_{b}+\lambda N_{b 0}+\lambda^{2} N_{b 1}\right)\right\rfloor_{6} v^{a} v^{b}, \\
& b_{5}=\frac{1}{120}\left\lfloor\lambda^{3}\left(N_{00}+\lambda N_{01}\right)^{3},\left(M_{a}+\lambda N_{a 0}+\lambda^{2} N_{a 1}\right)\left(M_{b}+\lambda N_{b 0}+\lambda^{2} N_{b 1}\right)\right\rfloor_{10} v^{a} v^{b}, \\
& b_{6}=\frac{1}{720}\left\lfloor\lambda^{4}\left(N_{00}+\lambda N_{01}\right)^{4},\left(M_{a}+\lambda N_{a 0}+\lambda^{2} N_{a 1}\right)\left(M_{b}+\lambda N_{b 0}+\lambda^{2} N_{b 1}\right)\right\rfloor_{15} v^{a} v^{b},
\end{aligned}
$$

and:

$$
\begin{aligned}
& c_{1}=\left\lfloor\lambda\left(N_{a b 0}+\lambda N_{a b 1}\right)\right\rfloor_{1} v^{a} v^{b}, \quad c_{2}=\frac{1}{2}\left\lfloor\lambda^{2}\left(N_{00}+\lambda N_{01}\right),\left(N_{a b 0}+\lambda N_{a b 1}\right)\right\rfloor_{2} v^{a} v^{b}, \\
& c_{3}=\frac{1}{6}\left\lfloor\lambda^{3}\left(N_{00}+\lambda N_{01}\right)^{2},\left(N_{a b 0}+\lambda N_{a b 1}\right)\right\rfloor_{3} v^{a} v^{b}, \\
& c_{4}=\frac{1}{24}\left\lfloor\lambda^{4}\left(N_{00}+\lambda N_{01}\right)^{3},\left(N_{a b 0}+\lambda N_{a b 1}\right)\right\rfloor_{4} v^{a} v^{b} .
\end{aligned}
$$

Separating the terms of order $\lambda^{4}$, we obtain:

$$
\begin{aligned}
\frac{b_{2 a b}}{\lambda^{4}}= & \frac{1}{2}\left\lfloor N_{a 1} N_{b 1}\right\rfloor_{1}, \\
\frac{b_{3 a b}}{\lambda^{4}}= & \frac{1}{6}\left(\left\lfloor N_{00}, N_{a 0}, N_{b 1}\right\rfloor_{6}+\left\lfloor N_{01}, M_{a}, N_{b 1}\right\rfloor_{6}+\left\lfloor N_{01}, N_{a 0} N_{b 0}\right\rfloor_{3}\right), \\
\frac{b_{4 a b}}{\lambda^{4}}= & \frac{1}{24}\left(\left\lfloor\left(N_{00}\right)^{2}, M_{a}, N_{b 1}\right\rfloor_{12}+\left\lfloor\left(N_{00}\right)^{2}, N_{a 0} N_{b 0}\right\rfloor_{6}\right) \\
& \left.+\left\lfloor N_{00}, N_{01}, M_{a}, N_{b 0}\right\rfloor_{24}+\left\lfloor\left(N_{01}\right)^{2}, M_{a} M_{b}\right\rfloor_{6}\right), \\
\frac{b_{5 a b}}{\lambda^{4}}= & \frac{1}{120}\left(\left\lfloor\left(N_{00}\right)^{3}, M_{a}, N_{b 0}\right\rfloor_{20}+\left\lfloor\left(N_{00}\right)^{2}, N_{01}, M_{a} M_{b}\right\rfloor_{30}\right), \\
\frac{b_{6 a b}}{\lambda^{4}}= & +\frac{1}{720}\left\lfloor\left(N_{00}\right)^{4}, M_{a} M_{b}\right\rfloor_{15},
\end{aligned}
$$

and:

$$
\begin{aligned}
& \frac{c_{2 a b}}{\lambda^{4}}=\frac{1}{2}\left\lfloor N_{01}, N_{a b 1}\right\rfloor_{2}, \\
& \frac{c_{3 a b}}{\lambda^{4}}=\frac{1}{6}\left(\left\lfloor\left(N_{00}\right)^{2}, N_{a b 1}\right\rfloor_{3}+\left\lfloor N_{00}, N_{01}, N_{a b 0}\right\rfloor_{6}\right), \\
& \frac{c_{4 a b}}{\lambda^{4}}=\frac{1}{24}\left\lfloor\left(N_{00}\right)^{3}, N_{a b 0}\right\rfloor_{4},
\end{aligned}
$$


where the contraction with $v^{a} v^{b}$ has been factored out but taken into account when building new permutations.

We now compute the traces in $\gamma^{5}\left(b_{a b}\right)$ and $\gamma^{5}\left(c_{a b}\right)$ using the fact that every matrix operator commutes with $\gamma^{5}$. For the terms involving $\gamma^{5}\left(b_{a b}\right)$, we find:

$$
\begin{aligned}
& \operatorname{tr} \gamma^{5}\left\lfloor N_{a 1} N_{b 1}\right\rfloor_{1}=0, \quad \operatorname{tr} \gamma^{5}\left\lfloor N_{00}, N_{a 0}, N_{b 1}\right\rfloor_{6}=0, \\
& \operatorname{tr} \gamma^{5}\left\lfloor\left(N_{00}\right)^{2}, M_{a}, N_{b 1}\right\rfloor_{12}=0, \\
& \operatorname{tr} \gamma^{5}\left\lfloor\left(N_{00}\right)^{2}, N_{a 0} N_{b 0}\right\rfloor_{6}=0, \quad \operatorname{tr} \gamma^{5}\left\lfloor\left(N_{00}\right)^{3}, M_{a}, N_{b 0}\right\rfloor_{20}=0, \\
& \operatorname{tr} \gamma^{5}\left\lfloor\left(N_{00}\right)^{2}, N_{01}, M_{a} M_{b}\right\rfloor_{30}=0, \quad \operatorname{tr} \gamma^{5}\left\lfloor\left(N_{00}\right)^{4}, M_{a} M_{b}\right\rfloor_{15}=0 .
\end{aligned}
$$

The only non-zero contributions are:

$$
\begin{gathered}
\operatorname{tr} \gamma_{5}\left\lfloor N_{01}, M_{a}, N_{b 1}\right\rfloor_{6}=k_{a} k_{i}\left(24 e^{2} F_{\alpha \beta} F_{4 b} \epsilon^{\alpha \beta i 4}+8 i e \epsilon^{\alpha \beta i j}\left[F_{\alpha \beta}, D_{b} D_{j}\right]\right) \equiv \mathfrak{B}_{1 a b}, \\
\operatorname{tr} \gamma_{5}\left[N_{01}, N_{a 0} N_{b 0}\right]_{3}=-8 i e k_{a} k_{b}\left(F_{\mu v} D_{i} D_{j}+D_{i} D_{j} F_{\mu v}-D_{i} F_{\mu v} D_{j}\right) \epsilon^{\mu v i j} \\
-8 i e k_{a} k_{j}\left(\left\{F_{\mu v},\left[D_{i}, D_{b}\right]\right\}+D_{b} F_{\mu v} D_{i}-D_{i} F_{\mu v} D_{b}\right) \epsilon^{\mu v i j} \equiv \mathfrak{B}_{2 a b}, \\
\operatorname{tr} \gamma_{5}\left[\left(N_{01}\right)^{2}, M_{a} M_{b}\right]_{6}=16 k_{a} k_{b} e^{2} \mathbf{k}^{2} \epsilon^{\alpha \beta \mu v} F_{\alpha \beta} F_{\mu v} \\
+16 e^{2} k_{a} k_{b} k_{i} k_{j}\left(4 \epsilon^{\alpha \beta j 4} F_{\alpha \beta} F_{i 4}-2 F_{\alpha \beta} F_{i v} \epsilon^{\alpha \beta j v}\right) \equiv \mathfrak{B}_{3 a b}, \\
\operatorname{tr} \gamma_{5}\left[N_{00}, N_{01}, M_{a}, N_{b 0}\right]_{24}=32 e i\left(k_{a} k_{i} k_{b} k^{\mu}\right) \epsilon^{\alpha \beta k i}\left[D_{\mu} D_{k} F_{\alpha \beta}-F_{\alpha \beta} D_{k} D_{\mu}\right] \\
+32 e i\left(k_{a} k_{i} k_{b} k^{\mu}\right) \epsilon^{\alpha \beta k i}\left[D_{k} F_{\alpha \beta} D_{\mu}-D_{\mu} F_{\alpha \beta} D_{k}\right] \equiv \mathfrak{B}_{4 a b} .
\end{gathered}
$$

Here, we introduce the notation $\mathfrak{B}_{1 a b}, \mathfrak{B}_{2 a b}$, which contribute to the term $b_{3 a b}$ together with $\mathfrak{B}_{3 a b}, \mathfrak{B}_{4 a b}$, which contribute to the term $b_{4 a b}$.

For the terms involving $c_{a b}$, we have:

$$
\begin{gathered}
\operatorname{tr} \gamma^{5}\left\lfloor N_{01}, N_{a b 1}\right\rfloor_{2}=0, \quad \operatorname{tr} \gamma^{5}\left\lfloor\left(N_{00}\right)^{2}, N_{a b 1}\right\rfloor_{3}=0, \\
\operatorname{tr} \gamma^{5}\left\lfloor N_{00}, N_{01}, N_{a b 0}\right\rfloor_{6}=0, \quad \operatorname{tr} \gamma^{5}\left\lfloor\left(N_{00}\right)^{3}, N_{a b 0}\right\rfloor_{4}=0,
\end{gathered}
$$

and thus:

$$
\operatorname{tr} \gamma^{5} \frac{c_{a b}}{\lambda^{4}}=0
$$

In other words, at this stage, we have:

$$
\left(\frac{1}{\lambda^{4}} \operatorname{tr} \gamma^{5} e^{\mathbb{A}+\lambda \mathbb{B}}\right)_{a b}=\frac{1}{3 !}\left(\mathfrak{B}_{1 a b}+\mathfrak{B}_{2 a b}\right)+\frac{1}{4 !}\left(\mathfrak{B}_{3 a b}+\mathfrak{B}_{4 a b}\right) .
$$

The Integration over the Momentum

Recalling Equation (46), the last step in our calculation is the integration over the momentum in the expression:

$$
\alpha^{(2)}(x)=\int \frac{\mathrm{d}^{4} k}{(2 \pi)^{4}} e^{-k_{\mu} k_{\mu}}\left(1-\left(v^{a} k_{a}\right)^{2}\right)\left(\frac{1}{\lambda^{4}} \operatorname{tr} \gamma^{5} e^{\mathbb{A}+\lambda \mathbb{B}}\right) \equiv \frac{1}{16 \pi^{2}}\left(\Delta_{1}+\Delta_{2}\right),
$$

which includes the full contribution to the anomaly in quadratic order in $v^{a}$, after using Equations (103)-(106) together with Equation (110). Once again, we perform the integrals given in Equation (75). 
To begin with, we deal with the first term of the right-hand side of Equation (111), which yields the contribution in the term $\Delta_{1}$. The momentum integration provides the intermediate results:

$$
\begin{gathered}
\frac{1}{\pi^{2}} \int d^{4} k e^{-k_{\mu} k_{\mu}}\left(\frac{1}{3 !} \mathfrak{B}_{1 a b}\right)=+\frac{2}{3} i e \epsilon^{\alpha \beta a j}\left[F_{\alpha \beta}, D_{b} D_{j}\right]+2 e^{2} F_{\alpha \beta} F_{4 b} \epsilon^{\alpha \beta a 4}, \\
\frac{1}{\pi^{2}} \int d^{4} k e^{-k_{\mu} k_{\mu}}\left(\frac{1}{3 !} \mathfrak{B}_{2 a b}\right)=+\frac{2}{3} i e \delta_{a b}\left(i e F_{i j} F_{\mu v}+D_{i} F_{\mu v} D_{j}\right) \epsilon^{\mu v i j} \\
+\frac{2}{3} i e \epsilon^{\mu v a j}\left(\left\{F_{\mu v},\left[D_{j}, D_{b}\right]\right\}+D_{b} F_{\mu v} D_{j}-D_{j} F_{\mu v} D_{b}\right), \\
\frac{1}{\pi^{2}} \int d^{4} k e^{-k_{\mu} k_{\mu}}\left(\frac{1}{4 !} \mathfrak{B}_{3 a b}\right)=e^{2} \delta_{a b} \frac{3}{4} \epsilon^{\alpha \beta \mu v} F_{\alpha \beta} F_{\mu v} \\
+e^{2}\left(\frac{4}{3} \epsilon^{\alpha \beta b 4} F_{\alpha \beta} F_{a 4}-\frac{2}{3} F_{\alpha \beta} F_{a v} \epsilon^{\alpha \beta b v}\right), \\
\frac{1}{\pi^{2}} \int d^{4} k e^{-k_{\mu} k_{\mu}}\left(\frac{1}{4 !} \mathfrak{B}_{4 a b}\right)=-\frac{1}{3} i e \delta_{a b} \epsilon^{\mu v i j}\left[+i e F_{\mu v} F_{i j}+2 D_{i} F_{\mu v} D_{j}\right] \\
-\frac{2}{3} i e \epsilon^{\mu v a j}\left[F_{\mu \nu} D_{j} D_{b}-D_{b} D_{j} F_{\mu v}+D_{b} F_{\mu v} D_{j}-D_{j} F_{\mu v} D_{b}\right] .
\end{gathered}
$$

Combining the above equations and after a long but straightforward calculation, the potentially dangerous terms containing covariant derivatives $D_{i}$ either cancel or arrange themselves to produce the commutator $\left[D_{i}, D_{j}\right]=-i e F_{i j}$. We recall that terms proportional to $k^{\mu}$ in the integration, arising from Equation (106), do not contribute when $\mu=4$ due to the antisymmetry in that variable. Furthermore, we remark that we can interchange $a \leftrightarrow b$ since the result must be symmetric after the multiplication by $v^{a} v^{b}$.

From Equation (110), we obtain:

$$
\begin{array}{r}
\frac{1}{\pi^{2}} \int d^{4} k e^{-k_{\mu} k_{\mu}}\left(\frac{1}{\lambda^{4}} \operatorname{tr} \gamma^{5} e^{\mathbb{A}+\lambda \mathbb{B}}\right)_{a b}=e^{2} \delta_{a b}\left[\frac{3}{4} \epsilon^{\mu v \alpha \beta} F_{\mu v} F_{\alpha \beta}-\frac{1}{3} \epsilon^{\mu v i j} F_{\mu v} F_{i j}\right] \\
-\frac{2}{3} e^{2} F_{\alpha \beta} F_{b v} \epsilon^{\alpha \beta a v}+\frac{2}{3} e^{2} \epsilon^{\alpha \beta a j} F_{\alpha \beta} F_{j b}-\frac{2}{3} e^{2} \epsilon^{\alpha \beta a 4} F_{\alpha \beta} F_{b 4} .
\end{array}
$$

Using the relation:

$$
\epsilon^{\mu v i j} F_{\mu v} F_{i j}=\frac{1}{2} \epsilon^{\mu v \alpha \beta} F_{\mu v} F_{\alpha \beta}
$$

in the first line of (116), and separating into $v=4$ and $v=j$ the summation over $v$ in the first term of the second line, results in:

$$
\begin{aligned}
\Delta_{1} & =\frac{1}{\pi^{2}} \int d^{4} k e^{-k_{\mu} k_{\mu}}\left(\frac{1}{\lambda^{4}} \operatorname{tr} \gamma^{5} e^{\mathbb{A}+\lambda \mathbb{B}}\right)_{a b} v^{a} v^{b} \\
& =e^{2} \delta_{a b} v^{a} v^{b} \frac{7}{12} \epsilon^{\mu \nu \alpha \beta} F_{\mu v} F_{\alpha \beta}-\frac{4}{3} e^{2} \epsilon^{\alpha \beta i b} F_{\alpha \beta} F_{i a} v^{a} v^{b}-\frac{4}{3} e^{2} F_{i j} F_{a 4} \epsilon^{i j b 4} v^{a} v^{b} .
\end{aligned}
$$

The additional identity:

$$
v^{a} v^{b} F_{\alpha \beta} F_{i a} \epsilon^{\alpha \beta i b}=v^{a} v^{b} F_{i j} F_{4 a} \epsilon^{i j b 4}+\frac{1}{4}|\mathbf{v}|^{2} \epsilon^{\mu v \alpha \beta} F_{\mu v} F_{\alpha \beta}, \quad|\mathbf{v}|^{2}=\delta_{a b} v^{a} v^{b},
$$

yields the further simplification:

$$
\Delta_{1}=\frac{1}{\pi^{2}} \int d^{4} k e^{-k_{\mu} k_{\mu}}\left(\frac{1}{\lambda^{4}} \operatorname{tr} \gamma^{5} e^{\mathbb{A}+\lambda \mathbb{B}}\right)_{a b} v^{a} v^{b}=\frac{1}{4} e^{2}|\mathbf{v}|^{2} \epsilon^{\mu \nu \alpha \beta} F_{\mu \nu} F_{\alpha \beta} .
$$

The remaining contribution $\Delta_{2}$ in the expression (111) comes from the zeroth order expansion in $v^{a}$ of the exponential, which is: 


$$
\operatorname{tr} \gamma_{5}\left(e^{A+\lambda B}\right)=\frac{1}{2 !} \operatorname{tr} \gamma^{5}\left(\lambda^{2} N_{01}\right)^{2}=\lambda^{4} \frac{e^{2}}{2} \epsilon^{\mu \nu \alpha \beta} F_{\mu \nu} F_{\alpha \beta}
$$

giving:

$$
\Delta_{2}=-v^{a} v^{b} \frac{1}{\lambda^{4} \pi^{2}} \int d^{4} k e^{-k_{\mu} k_{\mu}}\left(k_{b} k_{a}\right) \frac{e^{2}}{2} \epsilon^{\mu v \alpha \beta} F_{\mu v} F_{\alpha \beta}=-|\mathbf{v}|^{2} \frac{e^{2}}{4} \epsilon^{\mu v \alpha \beta} F_{\mu v} F_{\alpha \beta} .
$$

Summarizing, the second order contribution in $v^{a}$ to $\alpha(x)=\left(\Delta_{1}+\Delta_{2}\right) /\left(16 \pi^{2}\right)$ is identically zero.

\section{Discussion and Conclusions}

We calculated the abelian axial anomaly in a Lorentz violating model for a particular case of the modification $\gamma^{\mu} \rightarrow \gamma^{\mu}+d^{\mu}{ }_{v} \gamma^{v} \gamma^{5}$ introduced in the fermionic sector of the SME [2,7]. Motivated by the continuum Hamiltonian of a tilted WSM, whose cones have opposite tilting, we considered the LIV parameter $d^{\mu}{ }_{v}=\delta_{i}^{\mu} \delta_{v}^{0} v^{i}$, where $v^{i}$ is the tilting parameter given by the microscopic structure of the material. Using the Fujikawa path integral approach [30], we identify the modified axial current $J_{5}^{\mu}$ and calculate the anomaly to second order in the tilting parameter. For a linear order in $v^{i}$, we find the rather unexpected gauge invariant result $\alpha^{(1)}(x) \sim e v^{j} \partial_{j} \partial_{i} F^{i 0}$, saying that the chiral current obtains the additional term $J_{5}^{(1) \mu}=e v^{j} \partial_{j} F^{\mu 0}$. Its contribution to the chiral charge $Q_{5}^{(1)}=$ $\int d^{3} x J_{5}^{(1) 0}$ is identically zero, which displays this term as an irrelevant contribution to the anomaly. Thus, we conclude that the first order correction to the anomaly is zero. For the second order correction, we also found a zero contribution, after a highly non-trivial cancellation of a variety of combinations of spacetime operators which live in matrix space. Then, to the order considered, our result in the Euclidean space is the standard one:

$$
\alpha(x)=\frac{e^{2}}{32 \pi^{2}} F_{\mu \nu} F_{\rho \sigma} \varepsilon^{\mu \nu \rho \sigma}
$$

yielding the well-known anomaly in Minkowski space:

$$
\partial_{\mu} J_{5}^{\mu}=-\frac{e^{2}}{16 \pi^{2}} F_{\mu \nu} F_{\rho \sigma} \varepsilon^{\mu \nu \rho \sigma}=\frac{e^{2}}{2 \pi^{2}} \mathbf{E} \cdot \mathbf{B} .
$$

We then comment on previous work reported in the literature dealing with the calculation of axial anomalies in the Lorentz invariance violating (LIV) case, emphasizing those which use the Fujikawa approach and consider the $d^{\mu}{ }_{v}$ contribution in Equation (5). First, we compare our calculation with that of Ref. [34], appropriately expanded to the second order, and which served as a motivation for the present endeavor. Even though we deal with the same system, and contrary to our case, the authors in this reference find non-zero corrections to the standard axial anomaly using the Fujikawa approach.

As one can see from our calculation, after discarding the irrelevant term proportional to $v^{j} \partial_{j} \partial_{i} F^{i 4}$, the cancellation of the linear and the quadratic contributions heavily rests upon the appearance of terms including the covariant derivatives $D_{\mu}$, which must come in the right combinations to finally produce a gauge invariant result, in accordance with the regularization employed. In our case, the terms $D_{\mu} D^{\mu}$ cancel, and those proportional to $D_{i} D_{j} F_{k l}, D_{i} F_{k l} D_{j}, F_{k l} D_{i} D_{j}$, for example, manage to yield gauge invariant contributions which are functions of the electromagnetic tensor. We take these facts as a strong support to the correctness of our evaluation. Such factors involving the covariant derivatives do not appear in Ref. [34], and we feel that this is due to an incorrect separation of the non-commuting terms in the exponential. In fact, the non-commuting term $\mathbb{A}=$ $-2 i \gamma^{i} \gamma^{4} \gamma^{5} v_{4}^{j} k_{j} k_{i}$ cannot be factored out as $e^{\mathbb{A}} e^{\lambda \mathbb{B}}$ in the full expression $e^{\mathbb{A}+\lambda \mathbb{B}}$, which is the starting point of the calculation. 
Another representative work is that of Ref. [35], which also considers the Fujikawa approach to calculate the LIV abelian axial anomaly for a particular case of the contribution $\Gamma^{\mu}$ in the SME. They impose the restriction $d_{v}^{\mu}=Q c^{\mu}{ }_{v}$ with the idea of keeping the modified Dirac algebra as close as possible to the Lorentz covariant case. Here, $Q$ is a constant number. To apply their calculation to our case requires expressing our generalized gamma matrix $\Gamma^{\mu}$ in terms of theirs, which amounts to solving the equation:

$$
\Gamma^{\mu}=\gamma^{\mu}+\delta_{i}^{\mu} v^{i} \gamma^{0} \gamma^{5}=\left(\delta_{v}^{\mu}+c^{\mu}{ }_{v}\right) \gamma^{\nu}\left(1+Q \gamma^{5}\right) .
$$

It is a direct calculation to show that:

$$
c^{\mu}{ }_{\nu}=0, \quad Q=\frac{1}{4} v^{i} \gamma_{i} \gamma^{0},
$$

thus yielding a matrix-valued $Q$ which lends inapplicable their method to our problem. Nevertheless, the conclusion in Ref. [35] is that the corresponding abelian axial anomaly is not sensitive to the terms that violate Lorentz and that it is given by the standard Lorentz invariant expression (124). The authors in Ref. [36] also employ the Fujikawa method for the calculation of the anomaly in the Lorentz violating case, but they set $d_{v}^{\mu}=0$ from the outset.

Turning to the perturbative approach, the work in Ref. [37] generalizes the standard triangle calculation to include the tensors $c_{v}^{\mu}$ and $d_{v}^{\mu}$ without any restriction. The author deals with the general case of non-abelian chiral theories. His conclusion is that the left and right chiral anomalies are independent of the LIV parameters $c_{v}^{u}$ and $d_{v}^{u}$, thus keeping the original form corresponding to the Lorentz covariant case. In particular, the abelian chiral anomaly would be still given by Equation (124).

The clash between Ref. [34] and the general result [37], together with the fact that the alternative methods employed (Fujikawa approach versus perturbative calculation) are not known to be equivalent in the full LIV case, was our main motivation to perform this independent calculation. Our conclusion is also that, to the order considered, the calculated abelian axial anomaly is insensitive to the LIV corrections.

It is important to emphasize that even though the anomaly turned out to be independent of the LIV modifications of the fermionic Hamiltonian describing a specific material, this cannot imply that the electromagnetic response of the material will also be independent on them. For example, the different electromagnetic response of untilted $\left(d_{v}^{\mu}=0\right)$ versus tilted $\left(d_{v}^{\mu} \neq 0\right)$ Weyl semimetals has been experimentally shown in Refs. [38,39].

In fact, the calculation of the effective action using a chiral rotation involving the corresponding Jacobian, which is related to the anomaly, is rather subtle-as clearly shown in Ref. [21] for the case of a WSM. We find it illuminating to briefly describe the main steps of the procedure. The starting point is the Lagragian density:

$$
\mathcal{L}=i \bar{\Psi}\left(\gamma^{\mu}\left(\partial_{\mu}-i e A_{\mu}\right)+i b_{\mu} \gamma^{\mu} \gamma^{5}\right) \Psi,
$$

where $b_{\mu}$ is a four-vector which describes the separation of the Weyl nodes in energymomentum space. Then, an infinitesimal chiral rotation $\delta \theta(x)=i \delta s \gamma_{5} \theta(x), 0 \leq s \leq 1$, with $\theta(x)=b_{\mu} x^{\mu}$ is implemented, which leads to:

$$
Z\left[A_{\mu}\right]=\int \mathcal{D} \bar{\Psi} \mathcal{D} \Psi \exp \left[\ln J^{A}+\int d^{4} x \bar{\Psi} i \gamma^{\mu}\left(\partial_{\mu}-i e A_{\mu}+b_{\mu}(1-\delta s) \gamma_{5}\right) \Psi\right] .
$$

Observe that this rotation introduces both the Jacobian $J^{A}$ together with the axial current in the term proportional to $\delta$ s. Then, the Jacobian is calculated, yielding:

$$
\ln J^{A}=-\delta s \int d^{4} x \theta(x) \frac{e^{2}}{16 \pi^{2}} \epsilon^{\mu v \alpha \beta} F_{\mu v} F_{\alpha \beta} .
$$


The final step is the integration of $\delta s$ from zero to one, which eliminates the term $b_{\mu}(1-\delta s) \gamma_{5}$, and introduces the anomaly contribution (129). This says we can trade the original microscopic information contained in $b_{\mu} \gamma^{\mu} \gamma_{5}$ by the additional macroscopic effective electromagnetic action originating from $\ln J^{A}$, thus defining the effective action. In other words, the contribution $\ln J^{A}$ from the Jacobian of the chiral rotation by itself is not necessarily the effective action, unless we are able to perform steps similar to those shown above.

A pending goal in this research would be to obtain the effective electromagnetic action in our approximation and also as a non-perturbative result in the tilting parameter $v^{i}$. This would be especially important in the context of Weyl semimetals, since the value of $v^{i}$ will not necessarily as small as it must be in the case of LIV in the SME. Furthermore, the case $|\mathbf{v}|=1$ is of special physical importance, as it corresponds to cones tilted parallel to the Fermi energy plane. This value is also relevant since it distinguishes the so-called type-I $(|\mathbf{v}|<1)$ from type-II Weyl semimetals $(|\mathbf{v}|>1)$, and it is the point at which the density of states diverges, requiring an additional regularization [16]. Only a non-perturbative approach in $v^{i}$ could probe strongly tilted WSMs. A more complete calculation of the effective action would also necessarily involve the incorporation of the separation of the nodes given by $b_{\mu}$ in momentum space.

Author Contributions: Both authors equally contributed to this work. Both authors have read and agreed to the published version of the manuscript.

Funding: This research was funded by the project DGAPA-UNAM IN103319. Furthermore, we aknowledge support from CONACyT (México) under the project Fordecyt-Pronaces/ $428214 / 2020$.

Acknowledgments: We thank A. Salvio for informative correspondence.

Conflicts of Interest: The authors declare no conflict of interest.

\section{Appendix A. Useful Relations}

Here, we collect some identities that we used in the course of the calculation. For the traces of the Euclidean Dirac matrices, we have:

$$
\begin{aligned}
& \operatorname{tr} \gamma^{5}=\operatorname{tr} \gamma^{5} \gamma^{\mu} \gamma^{v}=\operatorname{tr} \gamma^{5} \gamma^{\mu} \gamma^{v} \gamma^{\rho}=0, \\
& \operatorname{tr} \gamma^{\mu_{1}} \gamma^{\mu_{2}} \ldots \gamma^{\mu_{2 n+1}}=0, \quad \mu_{n}=1,2,3,4, \\
& \operatorname{tr} \gamma^{\mu} \gamma^{v}=4 \eta^{\mu \nu}, \\
& \operatorname{tr} \gamma^{\mu} \gamma^{v} \gamma^{\rho} \gamma^{\sigma}=4\left(\eta^{\mu v} \eta^{\rho \sigma}+\eta^{\mu \sigma} \eta^{v \rho}-\eta^{\mu \rho} \eta^{v \sigma}\right), \\
& \operatorname{tr} \gamma^{5} \gamma^{\mu} \gamma^{v} \gamma^{\rho} \gamma^{\sigma}=-4 \varepsilon^{\mu \nu \rho \sigma}, \\
& \operatorname{tr} \gamma^{5} \gamma^{\mu} \gamma^{v} \gamma^{\rho} \gamma^{\sigma} \gamma^{\alpha} \gamma^{\beta}=-4\left(\eta^{\mu v} \varepsilon^{\rho \sigma \alpha \beta}-\eta^{\mu \rho} \varepsilon^{v \sigma \alpha \beta}+\eta^{v \rho} \varepsilon^{\mu \sigma \alpha \beta}\right. \\
& \left.\quad+\eta^{\alpha \beta} \varepsilon^{\mu v \rho \sigma}+\eta^{\sigma \alpha} \varepsilon^{\mu v \rho \beta}-\eta^{\sigma \beta} \varepsilon^{\mu \nu \rho \alpha}\right) .
\end{aligned}
$$

For example, the last relation gives:

$$
\operatorname{tr} \gamma^{5} \gamma^{\rho} \gamma^{4} \gamma^{\mu} \gamma^{v} \gamma^{j} \gamma^{4} F_{\mu v}=-4\left(\eta^{\rho 4} F_{\mu \nu} \varepsilon^{\mu v j 4}+2 F_{4 i} \varepsilon^{\rho j i 4}\right),
$$

which appears when computing the trace of Equation (103).

Furthermore, some relations involving the electromagnetic field tensor are:

$$
\begin{aligned}
& F_{\mu v} F_{i \sigma} \varepsilon^{\mu v i \sigma}=\frac{3}{4} F_{\mu v} F_{\rho \sigma} \varepsilon^{\mu v \rho \sigma}, \quad F_{\mu v} F_{i j} \varepsilon^{\mu v i j}=\frac{1}{2} F_{\mu v} F_{\rho \sigma} \varepsilon^{\mu v \rho \sigma}, \\
& F_{\mu v} F_{i 4} \epsilon^{\mu v i 4}=\frac{1}{4} F_{\mu v} F_{\rho \sigma} \varepsilon^{\mu v \rho \sigma}, \quad k_{i} k^{\rho} \varepsilon^{\mu v i j} F_{j \rho}=k_{j} k_{i} \varepsilon^{\mu v j r} F_{i r}, \\
& F_{\mu v} v^{a} v^{b} F_{i a} \varepsilon^{\mu v i b}=F_{i j} F_{4 a} v^{a} v^{b} \varepsilon^{i j b 4}+\frac{1}{4}|\mathbf{v}|^{2} F_{\mu v} F_{\rho \sigma} \varepsilon^{\mu v \rho \sigma},
\end{aligned}
$$


where the $\rho=4$ component in the last expression of Equation (A4) does not contribute as it will integrate to zero.

\section{References}

1. Colladay, D.; Kostelecký, V.A. CPT violation and the standard model. Phys. Rev. D 1997, 55, 6760-6774. [CrossRef]

2. Colladay, D.; Kostelecký, V.A. Lorentz-violating extension of the standard model. Phys. Rev. D 1998, 58, 116002. [CrossRef]

3. Kostelecký, V.A.; Mewes, M. Electrodynamics with Lorentz-violating operators of arbitrary dimension. Phys. Rev. D 2009, 80, 015020. [CrossRef]

4. Kostelecký, V.A.; Mewes, M. Signals for Lorentz violation in electrodynamics. Phys. Rev. D 2002, 66, 056005. [CrossRef]

5. Kubo, R. Statistical-Mechanical Theory of Irreversible Processes. I. General Theory and Simple Applications to Magnetic and Conduction Problems. J. Phys. Soc. Jpn. 1957, 12, 570-586. [CrossRef]

6. Kubo, R. Statistiacl Mechanics; North-Holland Publishing Company: Amsterdam, The Netherlands, 1971 ; p. 366.

7. Kostelecký, V.A.; Lehnert, R. Stability, causality, and Lorentz and CPT violation. Phys. Rev. D. 2001, 63, 065008. [CrossRef]

8. Wan, X.; Turner, A.M.; Vishwanath, A.; Savrasov, S.Y. Topological semimetal and Fermi-arc surface states in the electronic structure of pyrochlore iridates. Phys. Rev. B 2011, 83, 205101. [CrossRef]

9. Huang, S.-M.; Xu, S.-Y.; Belopolski, I.; Lee, C.-C.; Chang, G.; Wang, B.; Alidoust, N.; Bian, G.; Neupane, M.; Zhang, C.; et al. A Weyl Fermion semimetal with surface Fermi arcs in the transition metal monopnictide TaAs class. Nat. Commun. $2015,6,7373$. [CrossRef]

10. Lv, B.Q.; Weng, H.; Fu, B.B.; Wang, X.P.; Miao, H.; Ma, J.; Richard, P.; Huang, X.C.; Zhao, L.X.; Chen, G.F.; et al. Experimental Discovery of Weyl Semimetal TaAs. Phys. Rev. X 2015, 5, 031013. [CrossRef]

11. Xu, S.-Y.; Belopolski, I.; Alidoust, N.; Neupane, M.; Bian, G.; Zhang, C.; Sankar, R.; Chang, G.; Yuan, Z.; Lee, C.-C.; et al. Discovery of a Weyl fermion semimetal and topological Fermi arcs. Science 2015, 349, 613-617. [CrossRef]

12. Yang, L.X.; Liu, Z.K.; Sun, Y.; Peng, H.; Yang, H.F.; Zhang, T.; Zhou, B.; Zhang, Y.; Guo, Y.F.; Rahn, M.; et al. Weyl semimetal phase in the non-centrosymmetric compound TaAs. Nat. Phys. 2015, 11, 728-732. [CrossRef]

13. Xu, S.-Y.; Belopolski, I.; Sanchez, D.S.; Neupane, M.; Chang, G.; Yaji, K.; Yuan, Z.; Zhang, C.; Kuroda, K.; Bian, G.; et al. Spin Polarization and Texture of the Fermi Arcs in the Weyl Fermion Semimetal TaAs. Phys. Rev. Lett. 2016, 116, 096801. [CrossRef]

14. Nielsen, H.B.; Ninomiya, M. The Adler-Bell-Jackiw anomaly and Weyl fermions in a crystal. Phys. Lett. B 1983, 130, 389-396. [CrossRef]

15. Trescher, M.; Sbierski, B.; Brouwer, P.W.; Bergholtz, E.J. Quantum transport in Dirac materials: Signatures of tilted and anisotropic Dirac and Weyl cones. Phys. Rev. B 2015, 91, 115135. [CrossRef]

16. Soluyanov, A.A.; Gresch, D.; Wang, Z.; Wu, Q.S.; Troyer, M.; Dai, X.; Bernevig, B.A. Type II Weyl Semimetals. Nature 2015, 527, 495-498. [CrossRef]

17. Fukushima, K.; Kharzeev, D.E.; Warringa, H.J. Chiral magnetic effect. Phys. Rev. D 2008, 78, 074033. [CrossRef]

18. Kim, H.-J.; Kim, K.-S.; Wang, J.F.; Sasaki, M.; Satoh, N.; Ohnishi, A.; Kitaura, M.; Yang, M.; Li, L. Dirac versus Weyl Fermions in Topological Insulators: Adler-Bell-Jackiw Anomaly in Transport Phenomena. Phys. Rev. Lett. 2013, 111, 246603. [CrossRef]

19. Burkov, A.A.; Balents, L. Weyl Semimetal in a Topological Insulator Multilayer. Phys. Rev. Lett. 2011, 107, 127205. [CrossRef]

20. Grushin, A.G. Consequences of a condensed matter realization of Lorentz violating QED in Weyl semi-metals. Phys. Rev. D 2012, 86,045001 . [CrossRef]

21. Zyuzin, A.A.; Burkov, A.A. Topological response in Weyl semimetals and the chiral anomaly. Phys. Rev. B 2012, 86, 115133. [CrossRef]

22. Goswami, P.; Terawi, S. Axionic field theory of (3+1)-dimensional Weyl semimetals. Phys. Rev. B 2013, 88, 245107. [CrossRef]

23. Yan, B.; Felser, C. Topological Materials: Weyl Semimetals. Annu. Rev. Condens. Matter Phys. 2017, 8, 337-354. [CrossRef]

24. Gao, H.; Venderbos, J.W.F.; Kim, Y.; Rappe, A.M. Topological Semimetals from first-principles. Annu. Rev. Mater. Res. 2019, 49, 153-183. [CrossRef]

25. Hosur, P.; Qi, X. Recent developments in transport phenomena in Weyl semimetals. C. R. Phys. 2013, 14, 857-870. [CrossRef]

26. Vazifeh, M.M.; Franz, M. Electromagnetic Response of Weyl Semimetals. Phys. Rev. Lett. 2013, 111, 027201. [CrossRef] [PubMed]

27. Adler, S.L. Axial-Vector Vertex in Spinor Electrodynamics. Phys. Rev. 1969, 177, 2426-2438. [CrossRef]

28. Bell. J.S.; Jackiw, R. A PCAC Puzzle: $\pi^{0} \rightarrow \gamma \gamma$ in the $\sigma$-Model. II Nuovo Cimento A 1969, 60, 47-61. [CrossRef]

29. Jackson, J.D. Classical Electrodynamics; John Wiley \& Sons, Inc.: New York, NY, USA, 1998; p. 556.

30. Fujikawa, K.; Suzuki, H. Path Integrals and Quantum Anomalies; Clarendon Press: Oxford, UK; New York, NY, USA, 2004; pp. 65, 92, 149.

31. Bertlmann, R.A. Anomalies in Quantum Field Theory; Clarendon Press: Oxford, UK, 1996.

32. Sharma, G.; Goswami, P.; Tewari, S. Chiral anomaly and longitudinal magnetotransport in type-II Weyl semimetals. Phys. Rev. B 2017, 96, 045112. [CrossRef]

33. Zyuzin, V.A. On the magnetotransport of Weyl semimetals due to chiral anomaly. Phys. Rev. B 2017, 95, 245128. [CrossRef]

34. Zhang, K.; Zhang, E.; Xia, M.; Gao, P.; Zhang, S. The axial anomaly in chiral tilted Weyl semimetals. Ann. Phys. 2018, 394, 1-16. [CrossRef]

35. Arias, P.; Falomir, H.; Gamboa, J.; Mendez, F.; Schaposnik, F.S. Chiral anomaly beyond Lorentz invariance. Phys. Rev. D. 2007, 76, 025019. [CrossRef] 
36. Scarpelli, A.P.B.; Mariz, T.; Nascimento, J.R.; Petrov, A.Y. On the anomalies in Lorentz-breaking theories. Int. J. Mod. Phys. A 2016, 31, 1650063. [CrossRef]

37. Salvio, A. Relaxing Lorentz invariance in general perturbative anomalies. Phys. Rev. D 2008, 78, 085023. [CrossRef]

38. Zhang, C.-L.; Xu, S.-Y.; Belopolski, I.; Yuan, Z.; Lin, Z.; Tong, B.; Bian, G.; Alidoust, N.; Lee, C.-C.; Huang, S.-M.; et al. Signatures of the Adler-Bell-Jackiw chiral anomaly in a Weyl fermion semimetal. Nat. Commun. 2016, 7, 10735. [CrossRef] [PubMed]

39. Lv, Y.-Y.; Li, X.; Zhang, B.-B.; Deng, W.; Yao, S.-H.; Chen, Y.-F.; Zhou, J.; Zhang, S.-T.; Lu, M.-H.; Zhang, L.; et al. Experimental observation of anisotropic Adler-Bell-Jackiw anomaly in type-II Weyl semimetal $\mathrm{WTe}_{1.98}$ crystals at the quasi classical regime. Phys. Rev. Lett. 2017, 118, 096603. [CrossRef] 